\title{
Reorganization of intermolecular interactions in the polymorphic phase transition of a prototypical dithiazolyl-based bistable material ${ }^{\pi}$
}

Tommaso Francese ${ }^{\mathrm{a}, \mathrm{b}}$, Fernando Mota ${ }^{\mathrm{a}}$, Mercè Deumala, Juan J. Novoa ${ }^{\mathrm{a}}$, Remco W.A. Havenith ${ }^{b, c}$, Ria Broerb, Jordi Ribas-Arino ${ }^{a}{ }^{*}$

a Departament de Ciència dels Materials i Química Física and IQTCUB, Universitat de Barcelona, Spain

b Theoretical Chemistry, Zernike Institute for Advance Materials, University of Groningen, The

Netherlands

c Stratingh Institute for Chemistry, University of Groningen, The Netherlands

\section{Abstract:}

The spin transitions undergone by several molecular crystals of dithiazolyl (DTA) radicals make this type of radicals promising candidates for future sensors and memory devices. Here, we present a systematic computational study of the intermolecular interactions existing in the two polymorphs of the neutral radical 1,3,5-trithia-2,4,6-triazapentalenyl in order to elucidate the origin of the difference in energy between those two polymorphs involved in its spin transition and to understand the crystal packing of this prototype of bistable materials. The $\pi-\pi$ interactions between radicals are the main driving force for the crystal packing of both polymorphs, which comprises $\pi$-stacks of radicals. Among the interstack interactions, the strongest ones are those mediated by six- and four-center S $\cdots \mathrm{N}$ bridges. The difference in energy between polymorphs, in turn, is mainly controlled also by the intrastack $\pi-\pi$ intermolecular interactions, and the interstack S $\cdots S$ contacts instead of the S $\cdots N$ contacts. Since the supramolecular motifs herein identified as important for the crystal packing and/or for the energy difference between polymorphs (and, thus, for the spin transition temperature) are common to other members of the DTA family, our results provide valuable information to understand better the structure and properties of other switchable DTA-based materials.

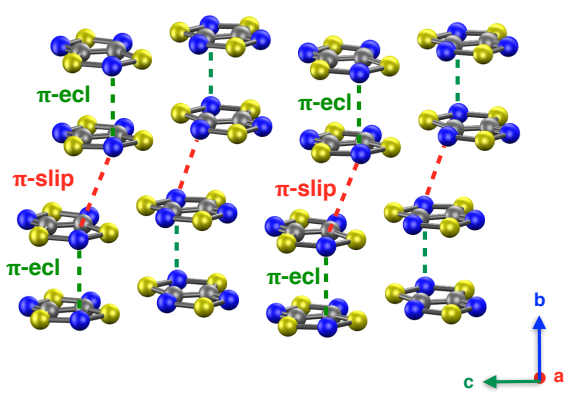

\footnotetext{
* Dr. Jordi Ribas-Arino

Departament de Ciència de Materials i

Química Física, Universitat de Barcelona and IQTCUB

c/Martí i Franquès 1, Barcelona, 08028

Spain

Phone: + 34934034864

E-mail: j.ribas@ub.edu
}

ॠ Dedicated to Jean-Paul Malrieu on the occasion of his 80th birthday, for his contribution to the modeling and the understanding of magnetic properties. 


\section{Reorganization of intermolecular interactions in the polymorphic phase transition of a prototypical dithiazolyl-based bistable materialף}

Tommaso Francese ${ }^{\mathrm{a}, \mathrm{b}}$, Fernando Mota ${ }^{\mathrm{a}}$, Mercè Deumala, Juan J. Novoa ${ }^{\mathrm{a}}$, Remco W.A. Havenith $^{\mathrm{b}, \mathrm{c}}$, Ria Broerb, Jordi Ribas-Arino ${ }^{\mathrm{a},{ }^{*}}$

a Departament de Ciència dels Materials i Química Física and IQTCUB, Universitat de Barcelona, Martí i Franquès 1, Barcelona, E-08028, Spain.

b Theoretical Chemistry, Zernike Institute for Advance Materials, University of Groningen, Nijenborgh 4, 9747 AG Groningen, The Netherlands

c Stratingh Institute for Chemistry, University of Groningen, 9747 AG Groningen, The Netherlands

$$
\text { E-mail: j.ribas@ub.edu }
$$

I Dedicated to Jean-Paul Malrieu on the occasion of his 80th birthday, for his contribution to the modeling and the understanding of magnetic properties.

\section{Abstract}

The spin transitions undergone by several molecular crystals of dithiazolyl (DTA) radicals make this type of radicals promising candidates for future sensors and memory devices. Here, we present a systematic computational study of the intermolecular interactions existing in the two polymorphs of the neutral radical 1,3,5-trithia-2,4,6-triazapentalenyl in order to elucidate the origin of the difference in energy between those two polymorphs involved in its spin transition and to understand the crystal packing of this prototype of bistable materials. The $\pi-\pi$ interactions between radicals are the main driving force for the crystal packing of both polymorphs, which comprises $\pi$-stacks of radicals. Among the interstack interactions, the strongest ones are those mediated by six- and four-center S $\cdots \mathrm{N}$ bridges. The difference in energy between polymorphs, in turn, is mainly controlled also by the intrastack $\pi-\pi$ intermolecular interactions, and the interstack $S \cdots S$ contacts instead of the $S \cdots N$ contacts. Since the supramolecular motifs herein identified as important for the crystal packing and/or for the energy difference between polymorphs (and, thus, for the spin transition temperature) are common to other members of the DTA family, our results provide valuable information to understand better the structure and properties of other switchable DTA-based materials. 


\section{Introduction}

Thiazyl-based radicals have been intensively investigated during the last decades because they can be used as versatile building blocks for molecular materials with interesting magnetic and/or electric properties. $1,2,3,4,5,6,7,8$ These radicals have also become main actors in the field of switchable molecular materials, i.e., materials whose physical properties can be altered by means of external stimuli, such as temperature and light.9,10,11 Indeed, the family of dithiazolyl (DTA) neutral radicals has furnished multiple examples of metal-free compounds capable of undergoing structural phase transitions that entail a drastic change in the magnetic response of the material when it is subjected to changes of temperature $12,13,14,15,16,17,18,19,20$, $21,22,23$ and, in some cases, when it is irradiated with light. ${ }^{19,24}$ It should be mentioned that the closely-related family of dithiadiazolyl radicals has also provided two examples of switchable magnetic materials in recent years. ${ }^{25,26}$ In some of the DTA phase transitions ${ }^{12-15,17,23}$, the transition temperature in the warming cycle is found to be higher than the transition temperature in the cooling cycle. This hysteretic behavior gives rise to a loop in the magnetic susceptibility versus temperature plot, yielding a temperature range of magnetic bistability wherein the crystal can be observed in two different states depending on its immediate history. This intriguing property, which can also be observed in transition-metal based spin-crossover compounds 27,28,29 and in other purely organic materials ${ }^{30,31,32}$, makes these radicals potential candidates for future sensors and memory devices. ${ }^{9,33}$

All the phase transitions of DTAs that have been reported so far involve the formation/cleavage of dimers of radicals. In many cases ${ }^{12-16,18,22}$, the dimers present in the crystal structure are cofacial $\pi$-dimers, which are held toghether by means of a long, multicenter bond ${ }^{34,35}$ (alternativaly called "pancake" bond $36,37,38$, ${ }^{39}$ ). These cofacial, eclipsed $\pi$-dimers are always found in the low-temperature (LT) phases of this family of switchable DTA compounds, where the cofacial, eclipsed $\pi$-dimers pile up giving rise to distorted $\pi$-stacks of DTA radicals that contain slipped pairs of cofacial $\pi$-dimers. Conversely, in the high-temperature (HT) phases of this family of switchable DTA compounds, the radicals pile up giving rise to regular $\pi$-stacks with a uniform distance between the slipped 
radicals. The distinct difference in magnetic response between the LT and HT phases (LT phases are typically diamagnetic, while the HT phases exhibit weak paramagnetism) originates in the large changes in the magnetic exchange couplings between adjacent radicals in the $\pi$-stacks upon phase transition. ${ }^{40,41}$

The transition temperature is a key property of any switchable material. Since the electronic energy ${ }^{42}$ difference between phases is one of the most important factors controlling the transition temperature, the identification of the elements that govern the energy difference between phases within a family of materials is a mandatory exercise in the quest of new materials with tailored transition temperatures. Here, we present a detailed computational study aimed at identifying the intermolecular interactions that play a key role in defining the energy difference between phases in switchable materials based on the formation/dissociation of eclipsed $\pi$-dimers between DTA radicals. As a model system of these materials, our study will focus on the 1,3,5-trithia-2,4,6triazapentalenyl (TTTA) neutral radical ${ }^{43}$, which has become a prototypical example of molecule-based bistable materials on account of its spin transition with a wide hysteresis loop encompassing room temperature (see Figure 1) that can be induced both with temperature and absorption of light $13,24,40,44,45,46,47,48,49,50,51,52$, $53,54,55$

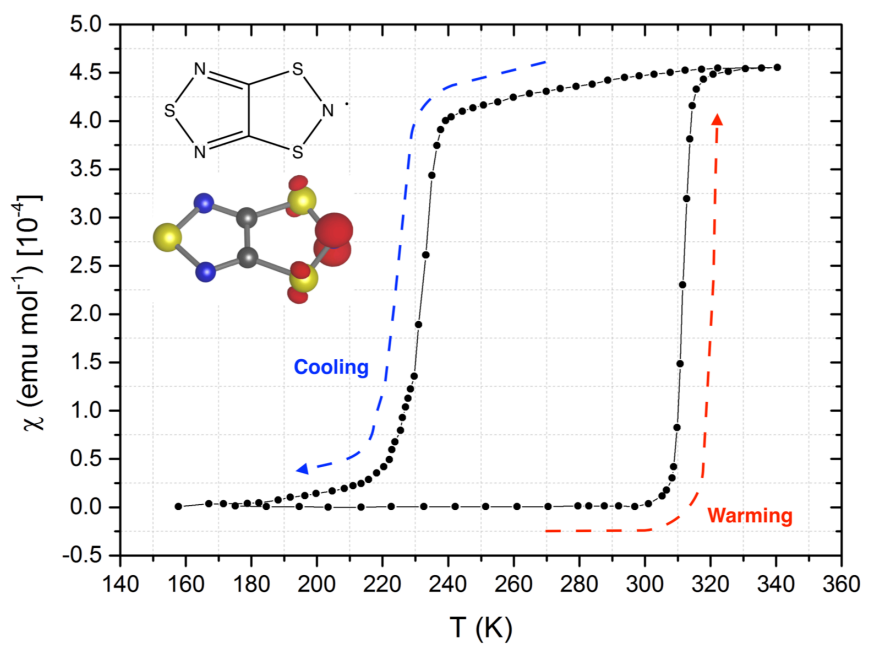

Figure 1. Temperature dependence of the paramagnetic susceptibility of the TTTA compound in the warming and cooling cycles. The insets show the molecular structure of a TTTA neutral radical and its spin density, which shows that the unpaired electron is delocalized over the S-N-S atoms of the dithiazolyl ring. 
The hysteretic phase transition of TTTA involves a LT diamagnetic phase and a HT paramagnetic phase. The triclinic ( $P \overline{1}$ space group) LT polymorph, which is the single polymorph observed on cooling below the bistability range, presents distorted $\pi$-stacks of radicals comprising slipped pairs of cofacial $\pi$-dimers. As displayed in Figure 2a, two types of intermolecular interactions between neighboring radicals are present in these distorted stacks: the long, multicenter bond between radicals in the eclipsed $\pi$-dimers (hereafter referred to as $\pi$-ecl interaction), and the interaction between slipped pairs of radicals ( $\pi$-slip interaction). Conversely, the monoclinic ( $P 2_{1} / c$ space group) HT polymorph, which is the single polymorph observed on heating above the bistability range, presents regular $\pi$-stacks of radicals, where each molecule exhibits a slipped overlap with its two adjacent molecules along the stacking direction ( $\pi$-slip interaction in Figure 3a).

(a)

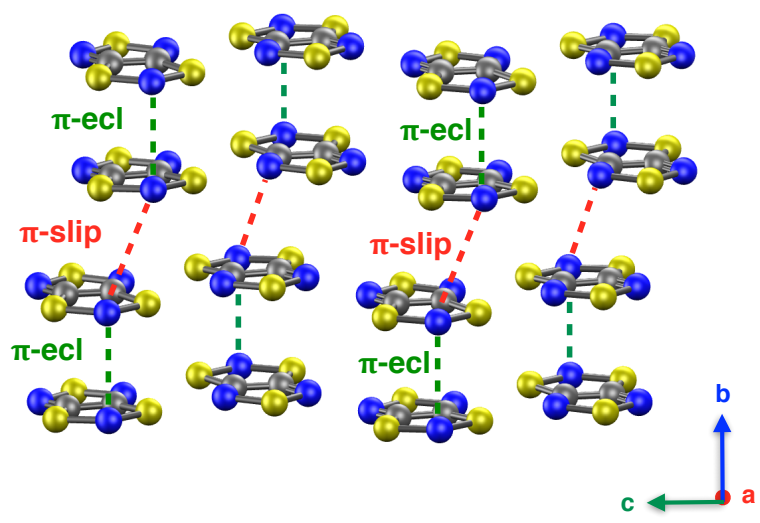

(c)

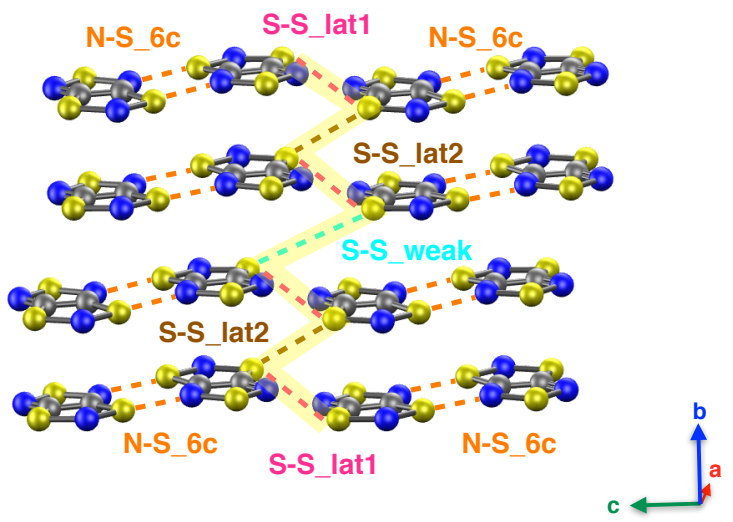

(b)

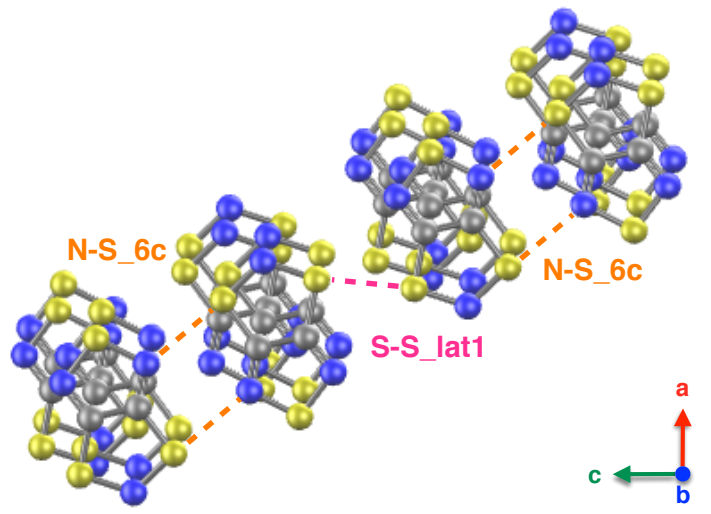

(d)

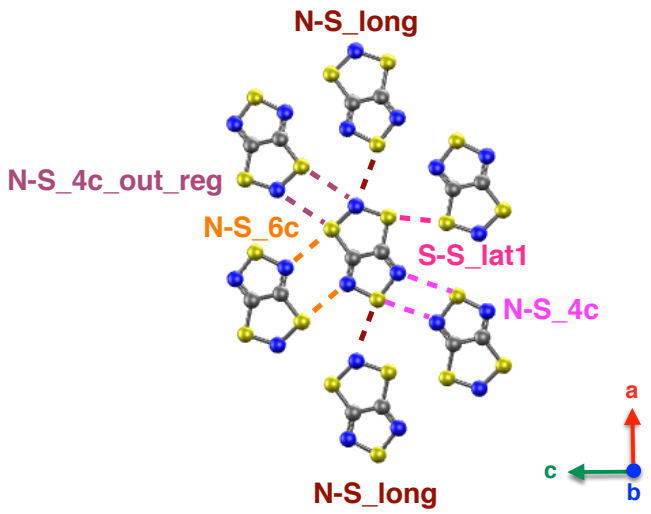

Figure 2. Different views of the crystal packing of the LT polymorph of TTTA (CCDC refcode $=$ SAXPOW06) at room temperature $(300 \mathrm{~K})$ along $(\mathrm{a}),(\mathrm{b}),(\mathrm{c})$ and (d) . The different types of intermolecular interactions between radicals are marked (see main text for contact nomenclature). 
(a)

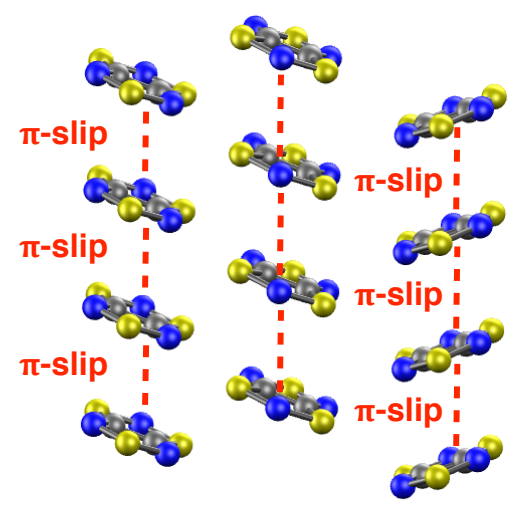

(c)

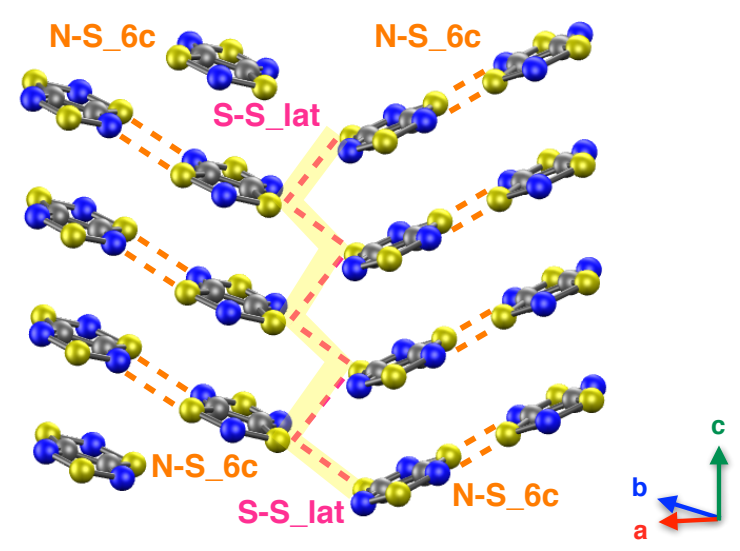

(b)
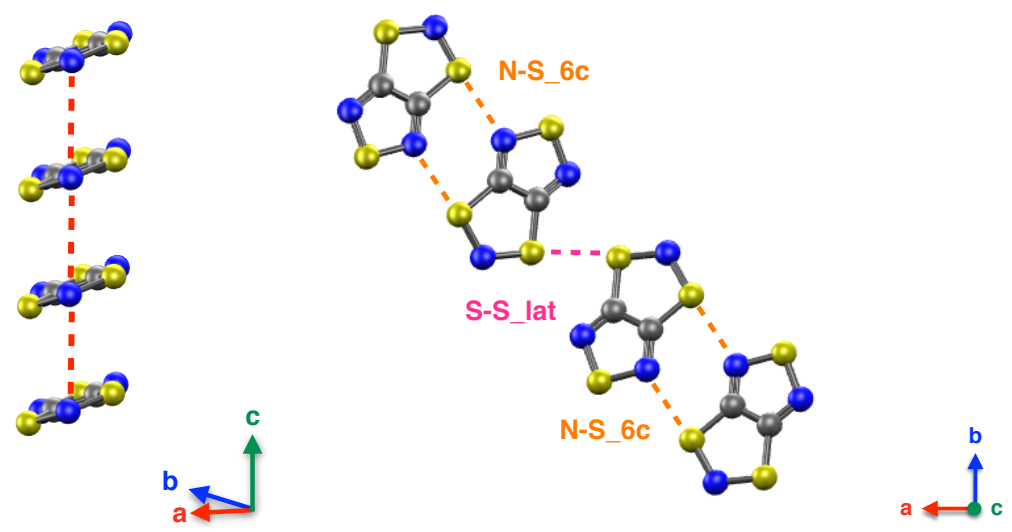

(d)

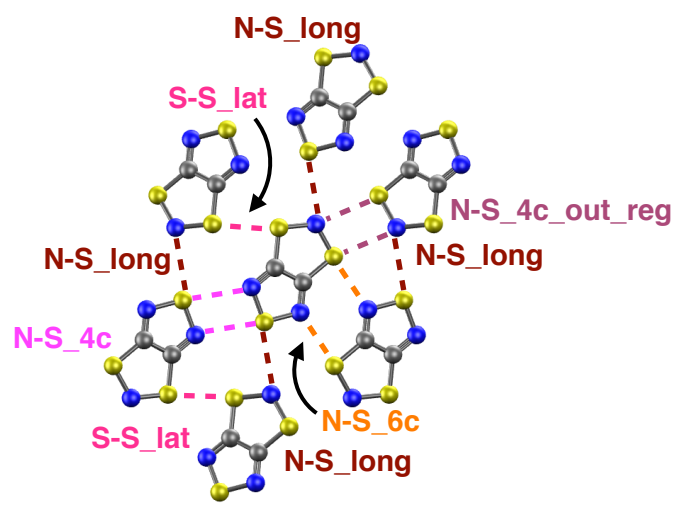

Figure 3. Different views of the crystal packing of the HT polymorph of TTTA (CCDC refcode $=$ SAXPOW05) at room temperature $(300 \mathrm{~K})$ along (a) , (b) , (c) and (d) . The different types of intermolecular interactions between radicals are marked (see main text for contact nomenclature).

The $\pi$-stacks of both LT and HT polymorphs of TTTA form 2D layers, where the $\pi$ stacks are laterally linked by a series of intermolecular 6 -center $\mathrm{S} \cdots \mathrm{N}$ bridges (which will be referred to as N-S_6c interactions) and intermolecular S...S contacts (S-S_lat interactions, see Figures $2 \mathrm{~b}$ and $3 \mathrm{~b}$ ). The 2D layers in LT contain one single molecular plane orientation (Figure 2c), whereas the 2D layers in HT contain two distinct molecular plane orientations (Figure 3c). The S-S_lat contacts in the 2D layers of both LT and HT phases define a zigzag pattern along the stacking direction ( $b$ axis) (Figures 2c and 3c). While the zigzag pattern in HT is regular (i.e., all the shortest interstack $S \cdots S$ contacts have the same distance), the zigzag pattern of LT features three different S-S_lat contacts: S-S_lat1, S-S_lat2 and S-S_weak, the 
latter having the longest S $\cdots S$ distance (see Figure 2c). The different 2D layers of both phases of the TTTA crystal are linked by a series of interstack contacts, as shown in Figures $2 \mathrm{~d}$ and $3 \mathrm{~d}$ : 4-center S...N bridges involving radicals in the sample plane (N-S_4c interactions), 4-center $\mathrm{S} \cdots \mathrm{N}$ bridges involving radicals of out-ofregistry stacks (N-S_4c_out_reg interactions), and S...N contacts (N-S_long interactions).

In this computational work, we will rationalize the different stability of the LT and HT polymorphs of TTTA on the basis of differences in intermolecular interactions between radicals. Specifically, the intermolecular interactions playing a leading role in establishing the energy difference between polymorphs and, thus, the transition temperature will be determined. In addition, we will identify which are the stronger intermolecular interactions and, thus, the interactions that drive the crystal packing of the polymorphs. Finally, the nature of the key intermolecular interactions present in the polymorphs will be evaluated. Since many of the intermolecular interactions herein considered are also present in other DTA-based crystals, the conclusions that will be drawn for TTTA will also be relevant for the other members of the family of DTA-based switchable materials.

\section{Computational details}

The analysis of the origin of the different stability of the two polymorphs of TTTA was performed by means of a set of single-point electronic structure calculations using different model systems: i) supercells containing 32 TTTA radicals for the solid-state calculations, ii) isolated $\pi$-stacks and isolated pairs of $\pi$-stacks of radicals, and iii) isolated dimers of radicals and isolated radicals. The single-point calculations of all these model systems were done using the relative atomic coordinates as directly extracted from the X-ray structures of the LT and HT polymorphs recorded at $300 \mathrm{~K} .^{13}$ These structures (whose diffraction data were collected using a Rigaku AFC5S four-circle diffractometer for HT, and on a Rigaku R-AXIS-IV imaging-plate system for LT) were obtained after isolating samples of the $\mathrm{HT}$ and LT phases by annealing at $40^{\circ} \mathrm{C}$ and at liquid nitrogen temperature for several hours, respectively. ${ }^{13}$ The supercells employed for the solid-state 
calculations include 8 stacks of radicals, each of them containing 4 radicals. The lattice parameters of these supercells are collected in Table 1. In the calculations of the isolated $\pi$-stacks and isolated pairs of $\pi$-stacks, each stack comprised 4 radicals and periodic boundary conditions were considered along the stacking direction (the supercell vectors associated with the stacking direction were the same ones as those employed in the solid-state calculations). In a recent work $^{54}$, we demonstrated that the regular $\pi$-stacking motif $(\cdots \mathrm{A} \cdots \mathrm{A} \cdots \mathrm{A} \cdots \mathrm{A} \cdots)_{\mathrm{n}}$ of the HT phase of TTTA is not a potential energy minimum but the average structure arising from a dynamic inter-conversion between two degenerate dimerized configurations: $(\cdots \mathrm{A}-\mathrm{A} \cdots \mathrm{A}-\mathrm{A} \cdots)_{\mathrm{n}} \leftrightarrow(-\mathrm{A} \cdots \mathrm{A}-\mathrm{A} \cdots \mathrm{A}-)_{\mathrm{n}}$. We also demonstrated that the regular $\pi-$ stacking motif is a minimum in the free energy surface of the system at $300 \mathrm{~K}$. Consequently, the regular stacks of the X-ray recorded structure of the HT polymorph properly represent this phase and are thus adequate for the analysis herein presented.

The single-point electronic structure studies for all model systems were carried out with plane wave pseudopotential calculations using the PBE exchangecorrelation functional ${ }^{56,57}$ within the spin unrestricted formalism, together with Vanderbilt ultrasoft pseudopotentials ${ }^{58}$, and $\Gamma$-point sampling of the Brillouin zone, as implemented in the QUANTUM ESPRESSO package. ${ }^{59}$ The semiempirical dispersion potential introduced by Grimme ${ }^{60}$ (D2 version) was added to the conventional Kohn-Sham DFT energy in order to properly describe the Van der Waals interactions between the different TTTA radicals. The plane wave basis set was expanded at a kinetic energy cutoff of $35 \mathrm{Ry}$.

Table 1. Supercell parameters employed in the calculations of the LT and HT polymorphs of TTTA. The $a, b$ and $c$ parameters are given in Angstrom. The $\alpha, \beta$ and $\gamma$ angles are given in degree.

\begin{tabular}{l|cccccc} 
& $a$ & $b$ & $c$ & $\alpha$ & $\beta$ & $\gamma$ \\
\hline LT $^{1}$ & 15.062 & 20.046 & 14.048 & 100.598 & 96.978 & 77.638 \\
HT & 18.888 & 14.844 & 15.063 & 90.0 & 104.628 & 90.0
\end{tabular}

1 The $(a, b, c)$ supercell parameters of LT and HT are multiples of the $(a, b, c)$ parameters defining the unit cells of the LT-300 and HT-300 X-ray resolved structures, respectively. The relations between the supercell (sc) parameters and the unit cell (uc) parameters are the following ones: $a_{\mathrm{LT}, \mathrm{sc}}=$ $2 \cdot a_{\mathrm{LT}, \mathrm{uc}} ; b_{\mathrm{LT}, \mathrm{sc}}=2 \cdot b_{\mathrm{LT}, \mathrm{uc}} ; c_{\mathrm{LT}, \mathrm{sc}}=2 \cdot c_{\mathrm{LT}, \mathrm{uc}} ; a_{\mathrm{HT}, \mathrm{sc}}=2 \cdot a_{\mathrm{HT}, \mathrm{uc}} ; b_{\mathrm{HT}, \mathrm{sc}}=4 \cdot b_{\mathrm{HT}, \mathrm{uc}} ; c_{\mathrm{HT}, \mathrm{sc}}=c_{\mathrm{HT}, \mathrm{uc}}$ 
Some of the plane wave pseudopotential calculations of isolated $\pi$-stacks and isolated pairs of $\pi$-stacks and the plane wave pseudopotential calculations of isolated pairs of radicals were benchmarked against all-electron PBE-D2 calculations with the cc-pVTZ basis set ${ }^{61,62}$ using the CRYSTAL09 code.63,64 The DFT calculations of isolated pairs of radicals, in turn, were validated by means of NEVPT2 $65,66 /$ aug-cc-pVTZ calculations using the ORCA code. ${ }^{67}$ These NEVPT2 calculations were carried out after CASSCF calculations using a minimum active space (i.e., 2 electrons in 2 orbitals). In all the calculations, an antiferromagnetic coupling between the spins of adjacent radicals was assumed, in agreement with previous studies. ${ }^{40,54}$

The interaction energy decomposition analysis performed for a selected subset of pairs of radicals was carried out using an Energy Decomposition Analysis ${ }^{68}$ method that can be applied within the DFT framework ${ }^{69}$, as implemented in the GAMESS suite of programs. ${ }^{70}$ In the method herein employed the interaction energy is decomposed into electrostatic, exchange, repulsion, polarization, and dispersion terms. ${ }^{69}$ The nature of the bonding between radicals was also evaluated using the "Atoms in Molecules" (AIM) methodology. ${ }^{71}$ Specifically, bond critical points (BCPs) between pairs of radicals were located with the AIMAll72 software, which allows for a fully automated analysis of the topological features of the Laplacian of the electron density distribution, including all critical points. The molecular wavefunction data required by the AIMAll code was obtained by means of calculations performed with Gaussian09.73

\section{Results and Discussion}

The presentation of the results is organized as follows. We will first analyze the intermolecular interactions in isolated pairs of radicals (Subsection 1). Then, we will investigate the different stability of the two polymorphs of TTTA on the basis of the energetics of isolated $\pi$-stacks of radicals and isolated pairs of $\pi$-stacks of radicals (Subsection 2). Finally, we will explore the nature of the intermolecular 
interactions that play a key role in determining the different stability of the two polymorphs of TTTA (Subsection 3).

\section{Intermolecular interactions in isolated pairs of TTTA radicals}

In this subsection, we analyze the interaction energy between radicals in different isolated pairs of TTTA radicals in order to i) identify the strongest intermolecular interactions in the TTTA polymorphs, i.e., the interactions that are key driving forces in the crystal packing, ii) provide insights into the origin of the different stability of the two phases of TTTA, and iii) validate the DFT methodology used in the calculations carried out with periodic boundary conditions (subsection 2).

The set of isolated pairs of radicals that have been considered for the LT and HT polymorphs are displayed in Figures 4 and 5, respectively. The results collected in
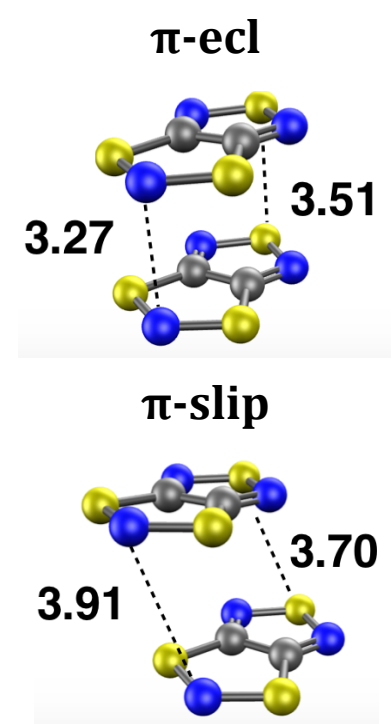

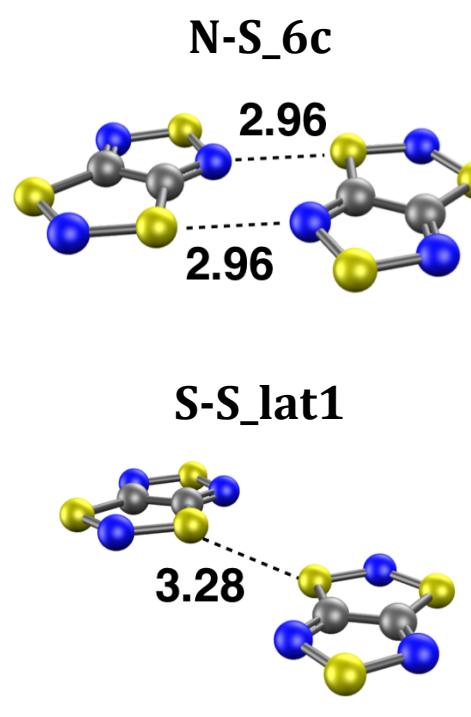

S-S_lat2

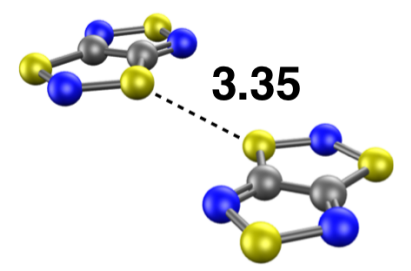

N-S_4c
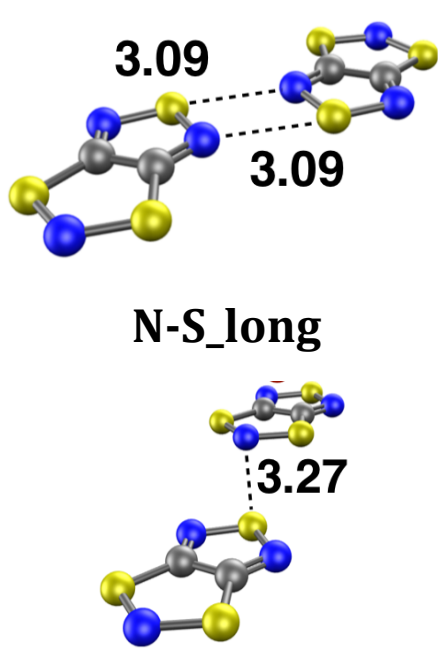

S-S_weak

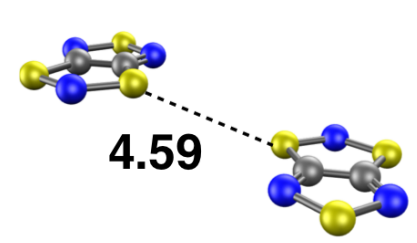

Figure 4. Different pairs of radicals in the LT polymorph of the TTTA crystal. The set of pairs considered in our study include those pairs with the shortest intermolecular contacts (distances are given in $\AA$ ). 

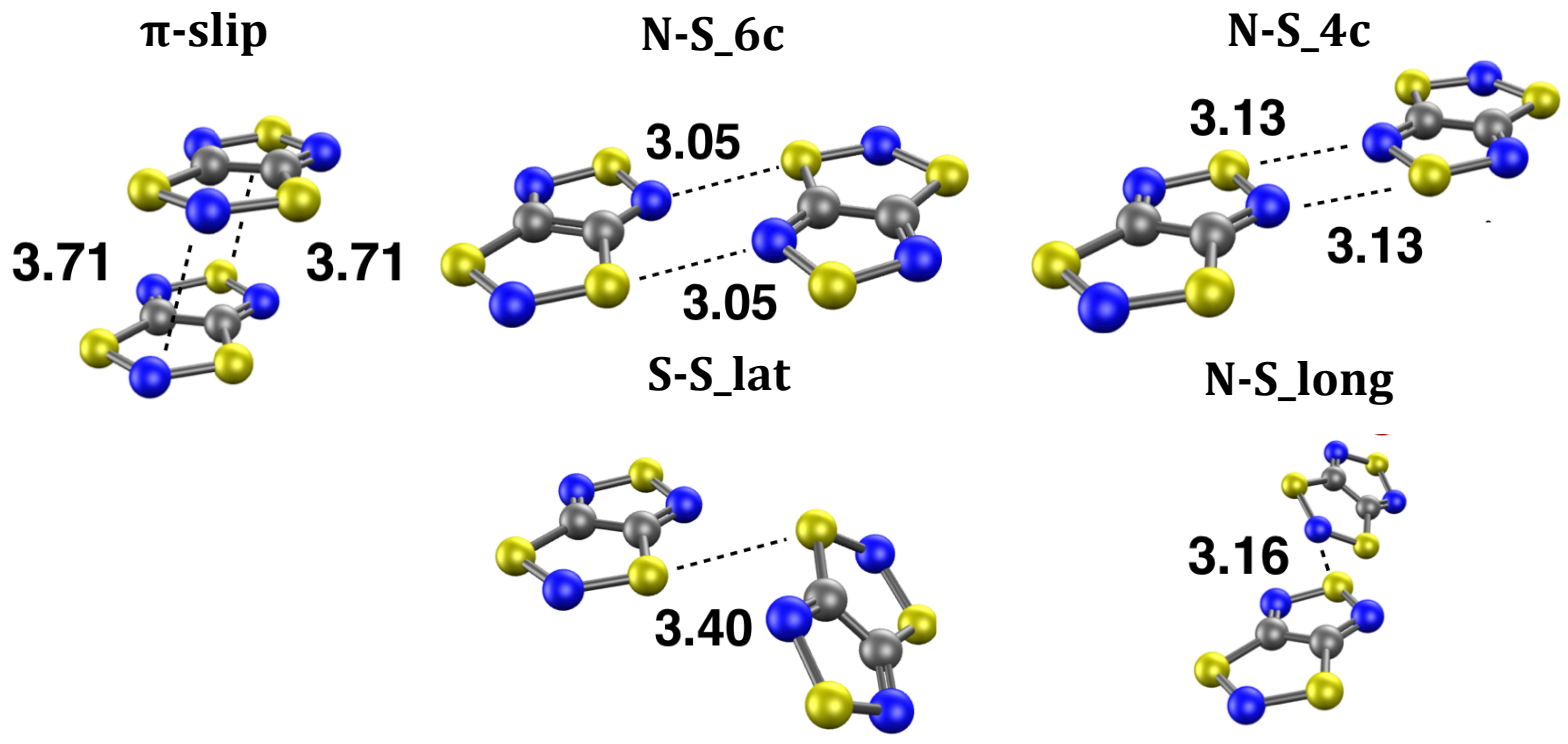

Figure 5. Different pairs of radicals in the HT polymorph of the TTTA crystal. The set of pairs considered in our study include those pairs with the shortest intermolecular contacts (distances are given in $\AA$ ).

Table 2. Interaction energies ${ }^{a}\left(E_{\text {int,LT }}\right.$ given in $\left.\mathrm{kcal} \mathrm{mol}^{-1}\right)$ between pairs of radicals in the LT polymorph of TTTA, ordered according to stability.

\begin{tabular}{|c|c|c|c|}
\hline Pair b & PBE-D2/USP-PWc & PBE-D2/cc-PVTZ ${ }^{\mathrm{d}}$ & NEVPT2/aug-cc-PVTZ \\
\hline$\pi-\mathrm{ecl}$ & -7.66 & -9.01 & -16.04 \\
\hline$\pi$-slip & -4.81 & -5.64 & -11.13 \\
\hline $\mathrm{N}-\mathrm{S} \_6 \mathrm{c}$ & -5.10 & -5.72 & -8.22 \\
\hline $\mathrm{N}-\mathrm{S} \_4 \mathrm{c}$ & -3.77 & -4.36 & -6.63 \\
\hline S-S_lat1 & -2.13 & -2.44 & -3.73 \\
\hline S-S_lat2 & -2.31 & -2.60 & -3.86 \\
\hline N-S_long & -1.35 & -1.67 & -2.81 \\
\hline S-S_weak & -0.57 & -0.73 & -0.98 \\
\hline
\end{tabular}

a Each interaction energy was computed as $E($ pair $)-E\left(\right.$ radical $\left._{1}\right)-E\left(\right.$ radical $\left._{2}\right)$, where $E($ pair $)$ is the energy of the pair of radicals and $E\left(\right.$ radical $\left._{1}\right)$ and $E\left(\right.$ radical $\left._{2}\right)$ are the energies of the isolated radicals forming the dimer; ${ }^{\mathrm{b}}$ The structures of the different types of pairs of radicals are displayed in Figure 4; cPlane wave pseudopotential DFT calculations carried out with Quantum Espresso; d All-electron DFT calculations using Gaussian basis sets carried out with Crystal; e Correlated wave function allelectron calculations carried out with ORCA.

Tables 2 and 3 reveal that the strongest intermolecular interaction between radicals in both polymorphs is found in the pairs exhibiting a $\pi-\pi$ interaction (be it either of the $\pi$-ecl or the $\pi$-slip type of interaction). It then follows that the intermolecular interactions along the $\pi$-stacks are the most attractive interactions in the crystals of TTTA. Among the other types of interaction between radicals (i.e. 
Table 3. Interaction energies $\left(E_{\text {int,HT }}\right.$ given in $\left.\mathrm{kcal} \mathrm{mol}^{-1}\right)$ between pairs of radicals in the HT polymorph of TTTA, ordered according to stability.

\begin{tabular}{|c|c|c|c|}
\hline Pairb & PBE-D2/USP-PW & PBE-D2/cc-PVTZd & NEVPT2/cc-PVTZ \\
\hline$\pi$-slip & -5.04 & -5.91 & -11.42 \\
\hline N-S_6c & -4.83 & -5.50 & -7.75 \\
\hline $\mathrm{N}-\mathrm{S} \_4 \mathrm{c}$ & -3.65 & -4.21 & -6.48 \\
\hline N-S_long & -1.61 & -1.94 & -3.31 \\
\hline S-S_lat & -2.24 & -2.54 & -4.05 \\
\hline
\end{tabular}

a Each interaction energy was computed as $E($ pair $)-E\left(\right.$ radical $\left._{1}\right)-E\left(\right.$ radical $\left._{2}\right)$, where $E($ pair $)$ is the energy of the pair of radicals and $E\left(\right.$ radical $\left._{1}\right)$ and $E\left(\right.$ radical $\left._{2}\right)$ are the energies of the isolated radicals forming the dimer; ${ }^{\mathrm{b}}$ The structures of the different types of pairs of radicals are displayed in Figure 5; c Plane wave pseudopotential DFT calculations carried out with Quantum Espresso; ${ }^{\mathrm{d}}$ All-electron DFT calculations using Gaussian basis sets carried out with Crystal; ${ }^{\mathrm{e}}$ Correlated wave function allelectron calculations carried out with ORCA.

Table 4. Difference in energies $\left(\Delta \mathrm{E}_{\text {int }}\left(\mathrm{HT}\right.\right.$-LT) given in $\left.\mathrm{kcal}^{\mathrm{mol}}{ }^{-1}\right)$ between pairs of TTTA radicals of the HT polymorph and pairs of radicals of the LT polymorph. ${ }^{\text {a }}$

\begin{tabular}{|c|c|c|c|c|}
\hline HT & LT & PBE-D2/USP-PWb & PBE-D2/cc-PVTZc & NEVPT2/cc-PVTZ \\
\hline$\pi$-slip & $\pi-\mathrm{ecl}$ & 2.58 & 2.96 & 4.77 \\
\hline$\pi$-slip & $\pi$-slip & -0.27 & -0.41 & -0.14 \\
\hline N-S_6c & $\mathrm{N}-\mathrm{S} \_6 \mathrm{c}$ & 0.24 & 0.09 & 0.63 \\
\hline N-S_4c & N-S_4c & 0.09 & 0.02 & 0.31 \\
\hline S-S_lat & S-S_lat1 & -0.14 & -0.21 & -0.13 \\
\hline S-S_lat & S-S_lat2 & 0.04 & -0.05 & -0.03 \\
\hline S-S_lat & S-S_weak & -1.70 & -1.94 & -2.91 \\
\hline N-S_long & N-S_long & -0.29 & -0.40 & -0.34 \\
\hline
\end{tabular}

a The difference in energy between two different pairs (pair1 and pair2) was computed as: $\Delta E$ (pair1, pair2) $=E$ (pair1, HT) $-E$ (pair2, LT); b Plane wave pseudopotential DFT calculations carried out with Quantum Espresso; c All-electron DFT calculations using Gaussian basis sets carried out with Crystal; ${ }^{\mathrm{d}}$ Correlated wave function all-electron calculations carried out with ORCA.

the interactions that connect $\pi$-stacks), the N-S_6c and N-S_4c interactions are significantly stronger than the others.

According to the differences in energy between pairs of radicals gathered in Table 4, the different stability of the LT and HT phases of TTTA is mainly controlled by the two following types of interaction: the interactions along the $\pi$-stacks $\left(\pi\right.$-slip ${ }^{\mathrm{HT}}$ and $\pi-e c l^{\mathrm{LT}}$ ) and the interstack S..S contacts (S-S_lat ${ }^{\mathrm{HT}}$ and S-S_weak ${ }^{\mathrm{LT}}$ ). The former type of interactions favor the LT polymorph because the transformation of the $\pi$ ecl interactions into $\pi$-slip interactions in going from LT to HT results in a much less attractive interaction ( $\sim 4.7 \mathrm{kcal} \mathrm{mol}^{-1}$, according to NEVPT2 calculations) and, 
thus, in a lower stability of HT. The interstack S...S contacts, instead, favor the HT polymorph due to the disruption of the regular zigzag pattern of S...S contacts in

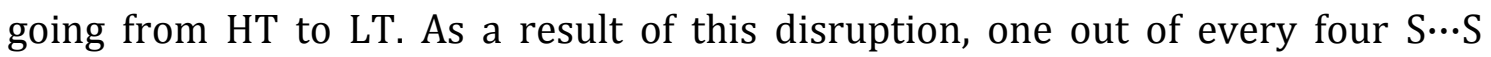
contacts increases its distance (i.e., one out of every four S-S_lat ${ }^{\mathrm{HT}}$ interactions transforms itself into a S-S_weak ${ }^{\mathrm{LT}}$ interaction), thereby decreasing significantly its associated interaction energy (by about $2.9 \mathrm{kcal} \mathrm{mol}^{-1}$, according to NEVPT2 calculations).

The results collected in Tables 2, 3 and 4 also demonstrate that PBE-D2 calculations (both with plane waves and cc-PVTZ basis sets) properly capture the main trends observed in the more accurate NEVPT2 calculations. Although the interaction energies and the differences in interaction energy obtained at the PBED2 level are smaller than those obtained at the NEVPT2 level, the conclusions that can be drawn from the PBE-D2 calculations are fully consistent with the conclusions drawn from the NEVPT2 calculations. It thus follows that the PBE-D2 methodology is adequate for describing the intermolecular interactions between TTTA radicals and, thus, adequate for the calculations considering periodic boundary conditions that will be presented in the next subsection.

\section{Intermolecular interaction model systems considering periodic boundary conditions}

In this subsection we shall further investigate the origin of the different stability of the two polymorphs of TTTA by means of PBE-D2 calculations of isolated $\pi$-stacks and isolated pairs of $\pi$-stacks.

Let us set the stage by first establishing the difference in lattice cohesive energy ${ }^{74}$ between the two phases of TTTA. According to plane wave pseudopotential PBED2 solid-state calculations carried out using supercells of 32 radicals, the LT phase of TTTA is $0.92 \mathrm{kcal} \mathrm{mol}^{-1}$ more stable (given per TTTA molecule) than the HT phase ( $c f$. Table 5). The agreement between this value and the transition enthalpy of $0.6 \mathrm{kcal} \mathrm{mol}^{-1}$ measured in DSC experiments ${ }^{45}$ is remarkable taking into account that we are dealing with very small energy differences. The error associated with 
Table 5. Differences in energy, $\Delta E$ (given in $\mathrm{kcal} \mathrm{mol}^{-1}$ ), between the HT and LT polymorphs of TTTA and between an isolated $\pi$-stack of HT and an isolated $\pi$-stack of LT.

\begin{tabular}{c|c}
$\Delta E$ polymorphs & $\Delta E$ isolated $\pi$-stacks \\
\hline 0.92 & $1.08^{\mathrm{b}}$
\end{tabular}

\footnotetext{
a The differences in energy are calculated as $E(\mathrm{HT})-E(\mathrm{LT})$, and are given per TTTA radical. The values were obtained by means of plane wave pseudopotential DFT calculations carried out with Quantum Espresso.

b This value, obtained with Quantum Espresso, was further corroborated with all-electron calculations using a cc-pVTZ basis set (carried out with CRYSTAL09), which furnished a very similar difference in energy of $1.27 \mathrm{kcal} \mathrm{mol}^{-1}$.
}

the computational estimation of lattice energies is commonly $1 \mathrm{kcal} \mathrm{mol}^{-1}$ or slightly above ${ }^{75}$. Nevertheless, the computational estimation of differences between the lattice energies of two polymorphs of TTTA within the framework of the same level of theory is perfectly meaningful.

The calculations carried out on isolated $\pi$-stacks, in turn, show that an isolated $\pi$ stack of LT is $1.08 \mathrm{kcal} \mathrm{mol}^{-1}$ more stable than an isolated $\pi$-stack of HT. The fact that these two values are so similar demonstrates that the intra- $\pi$-stack interactions are the leading factor behind the different stability of the two polymorphs of TTTA. In fact, the difference in energy between a $\pi$-stack of LT and a $\pi$-stack of HT can be accurately predicted on the basis of the differences in energy gathered in Table 4. If we assume that the energy of a given column (given per radical) is determined exclusively by the energy of the TTTA radical and the sum of interaction energies between this radical and its nearest neighbors, the expression of the energies of the $\pi$-stacks of LT and HT can be written as:

$$
\begin{aligned}
& E_{\text {stack }}^{L T}=E_{\text {rad }}+\frac{1}{2} E_{\text {int }}^{\pi-e c l}+\frac{1}{2} E_{i n t}^{\pi-s l i p, L T} \\
& E_{\text {stack }}^{H T}=E_{\text {rad }}+E_{\text {int }}^{\pi-s l i p, H T}
\end{aligned}
$$

where $E_{\text {rad }}$ is the the energy of a single radical and $E_{\text {int }}$ refers to the interaction energies between radicals. Note that in the expressions given above we distinguish the $\pi$-interaction between slipped radicals in LT from that of HT because they do not present the same interaction energy ( $c f$. Tables 2, 3 and 4). On the basis of Eqs. 
1 and 2, the difference in energy between an isolated $\pi$-stack of HT and isolated $\pi$ stack of LT can be expressed as:

$E_{\text {stack }}^{H T}-E_{\text {stack }}^{L T}=\frac{1}{2}\left(E_{\text {int }}^{\pi-s l i p, H T}-E_{\text {int }}^{\pi-e c l}\right)+\frac{1}{2}\left(E_{\text {int }}^{\pi-s l i p, H T}-E_{\text {int }}^{\pi-s l i p, L T}\right)$

The difference in energy between the HT and LT $\pi$-stacks that results from inserting the values of the first two entries of the "PBE-D2/USP-PW" column of Table 4 in the equation above is $1.15 \mathrm{kcal} \mathrm{mol}^{-1}$. The close correspondence between this value and the value obtained from the PBE-D2 calculations of isolated $\pi$-stacks $\left(1.08 \mathrm{kcal} \mathrm{mol}^{-1}\right)$ demonstrates that the difference in energy between the HT and LT isolated $\pi$-stacks is essentially governed by the short-range two-body interactions between neighboring radicals, primarily by the difference in energy between the $\pi$-ecl dimers of LT and the $\pi$-slip dimers of HT.

Upon comparing the difference in lattice energy between the HT and LT polymorphs (0.92 kcal mol-1) with the difference in energy between the HT and LT isolated $\pi$-stacks $\left(1.08 \mathrm{kcal} \mathrm{mol}^{-1}\right)$, it is concluded that the former is largely dominated by the latter and that the interactions between $\pi$-stacks make a nonnegligible contribution to the energy gap between HT and LT, specifically by decreasing it. In view of the non-negligible contribution of the interstack interactions, we shall now study them in detail. The interaction energies between $\pi$-stacks in both HT and LT polymorphs ( $c f$. Table 6) follow the same trend as that observed in the interaction energies for the isolated pairs of radicals (Tables 2 and $3)$. Indeed, the strongest interaction between $\pi$-stacks is found for the stacks that interact through the $\mathrm{N}-\mathrm{S} \_6 \mathrm{c}$ interactions, which feature the largest interaction energy among the interstack contacts. The other interstack contacts are, in decreasing order of interaction strength, those mediated by N-S_4c contacts, lateral S...S contacts and N-S_long contacts ( $c f$. Table 6). As can be seen in Tables 2 and 3 , the interaction energies of isolated pairs of radicals have the same ordering. However, the values of the interaction energies between $\pi$-stacks in Table 6 are considerably larger than the values that would be predicted on the basis of the interaction energies of isolated pairs of radicals (note that the values of Table 6 are given per radical, while the values of Tables 2 and 3 are given per pairs of radicals). 
Table 6. Interaction energies $\left(E_{\text {int }}\right)$ and differences in interaction energies $\left(\Delta E_{\text {int }}\right)$ (given in kcal mol-1) between $\pi$-stacks of radicals in both HT and LT polymorphs.a

\begin{tabular}{l|c|c|c} 
Type of interaction & $E_{\text {int }}(\mathrm{HT})$ & $\mathrm{E}_{\text {int }}(\mathrm{LT})$ & $\Delta E_{\text {int }} \mathrm{d}$ \\
\hline N-S_6c & -3.46 & -3.34 & -0.11 \\
N-S_4c & -2.29 & -2.41 & 0.12 \\
S-S_latb & -2.13 & -1.86 & $-0.27 \mathrm{e}$ \\
N-S_long & -1.06 & -1.17 & 0.11 \\
N-S_4c_out_regc & -1.38 & -1.48 & 0.10
\end{tabular}

a The interaction energies are given per TTTA radical. The values reported were obtained by means of plane wave pseudopotential DFT calculations carried out with Quantum Espresso.

${ }^{b}$ Note that the S-S_lat-type interaction in HT involves only one type of interaction between pairs of radicals (the S-S_lat interaction, see Fig. 3c). Conversely, the S-S_lat-type interaction in LT involves three different types of interaction between radical pairs, namely: S-S_lat1, S-S_lat2 and S-S_weak (see Figure 2c).

c The "N-S_4C_out_reg" type of interaction present in these columns resembles the type of interaction present in the "N-S_4c" case. However, in the former case the $\pi$-stacks are out-ofregistry, whereas in the latter they are in-registry (see Figures $2 \mathrm{~d}$ and $3 \mathrm{~d}$ ).

$\mathrm{d}$ For a given type of interaction, the difference in interaction energy $\left(\Delta E_{\text {int }}\right)$ was obatined via:

$\Delta E_{\text {int }}=E_{\text {int }}(\mathrm{HT})-E_{\text {int }}(\mathrm{LT})$.

e This value, obtained with Quantum Espresso, was further corroborated with all-electron calculations using a cc-pVTZ basis set (carried out with CRYSTAL09), which furnished a very similar difference in interaction energy of $-0.30 \mathrm{kcal} \mathrm{mol}^{-1}$.

This implies that the interaction energy between $\pi$-stacks cannot be expressed solely in terms of a sum of pairwise interaction energies between nearest neighbors, contrary to the intra- $\pi$-stack interaction energies.

When considering the difference in interaction energies between the $\pi$-stacks of HT and the $\pi$-stacks of LT ( $c f$. Table 6), it is observed that all types of interactions result in small but not negligible relative stabilizations of either one or the other polymorph. Among all types of lateral interactions, the lateral S $\cdots S$ contacts are those that lead to a larger stabilization of one polymorph relative to the other one. Specifically, the lateral S $\cdots S$ contacts lead to a significant relative stabilization of the HT polymorph because the interaction energy between $\pi$-stacks through this type of contacts at HT is $0.27 \mathrm{kcal} \mathrm{mol}^{-1}$ more stable than at LT. This result is in line with the intermolecular interactions evaluated for isolated pairs of radicals (see previous subsection), which showed that the increase of the S...S distance in going from the S-S_lat interaction at HT to the S-S_weak interaction at LT entailed a considerable weakening (by $0.85 \mathrm{kcal} \mathrm{mol}^{-1}$, cf. Table 4$)^{76}$ of the intermolecular 
interaction between radicals. It should be mentioned, though, that the relative stabilization of $0.27 \mathrm{kcal} \mathrm{mol}^{-1}$ is somewhat smaller than the value that might have been expected on the basis of the results obtained for the isolated pairs. As explained in the previous paragraph, this is due to the fact that the interaction energy between $\pi$-stacks cannot be expressed solely in terms of a sum of pairwise interaction energies between nearest neighbors.

\section{Evaluation of the nature of the key intermolecular interactions}

In this subsection we shall gain further insight into the energetic differences between the LT and HT polymorphs of TTTA by means of an analysis of the intermolecular bond critical points (BCPs) present in the different pairs of radicals and an energy decomposition analysis of the interaction energy for the most relevant pairs. Before presenting the results of these analyses, it is worth mentioning that the intermolecular interactions in the crystal induce very small changes in the electron density of a TTTA radical, as shown by the electron density difference isosurfaces shown in Figure 6. The deformation of the electron density, which is mainly localized in the S-N-S fragment of the dithiazolyl ring, is slightly larger in the LT polymorph than in the HT polymorph.
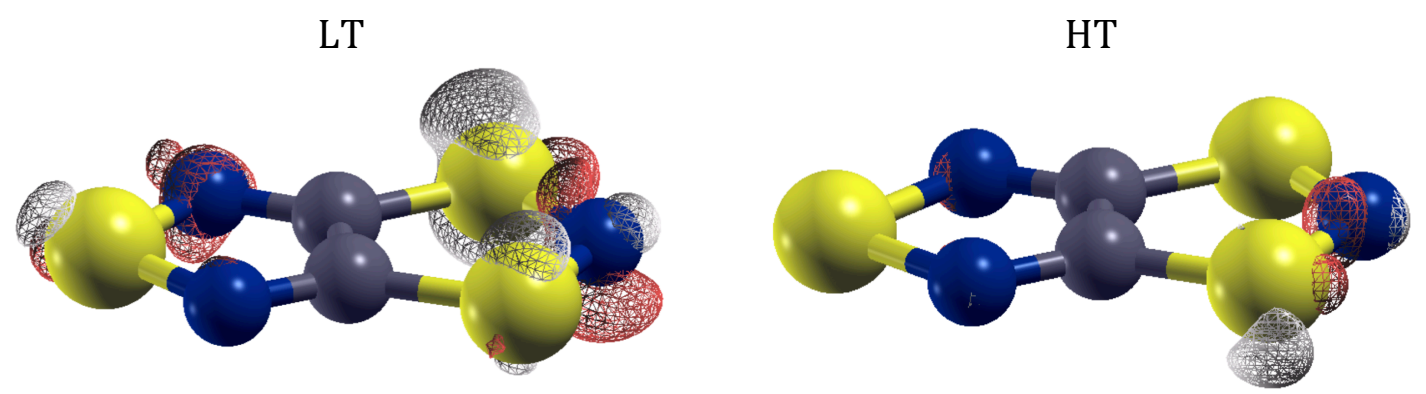

Figure 6. Electron density difference isosurfaces at an isovalue of $0.0015 e / \AA^{3}$ for a TTTA radical in the LT (left) and in the HT (right) polymorphs; grey/red isosurfaces indicate charge depletion/accumulation. The electron density differences have been computed by subtracting the electron density of a given TTTA radical in the solid state (either in the LT or HT polymorphs) and the electron density of the same TTTA radical in the gas phase. 

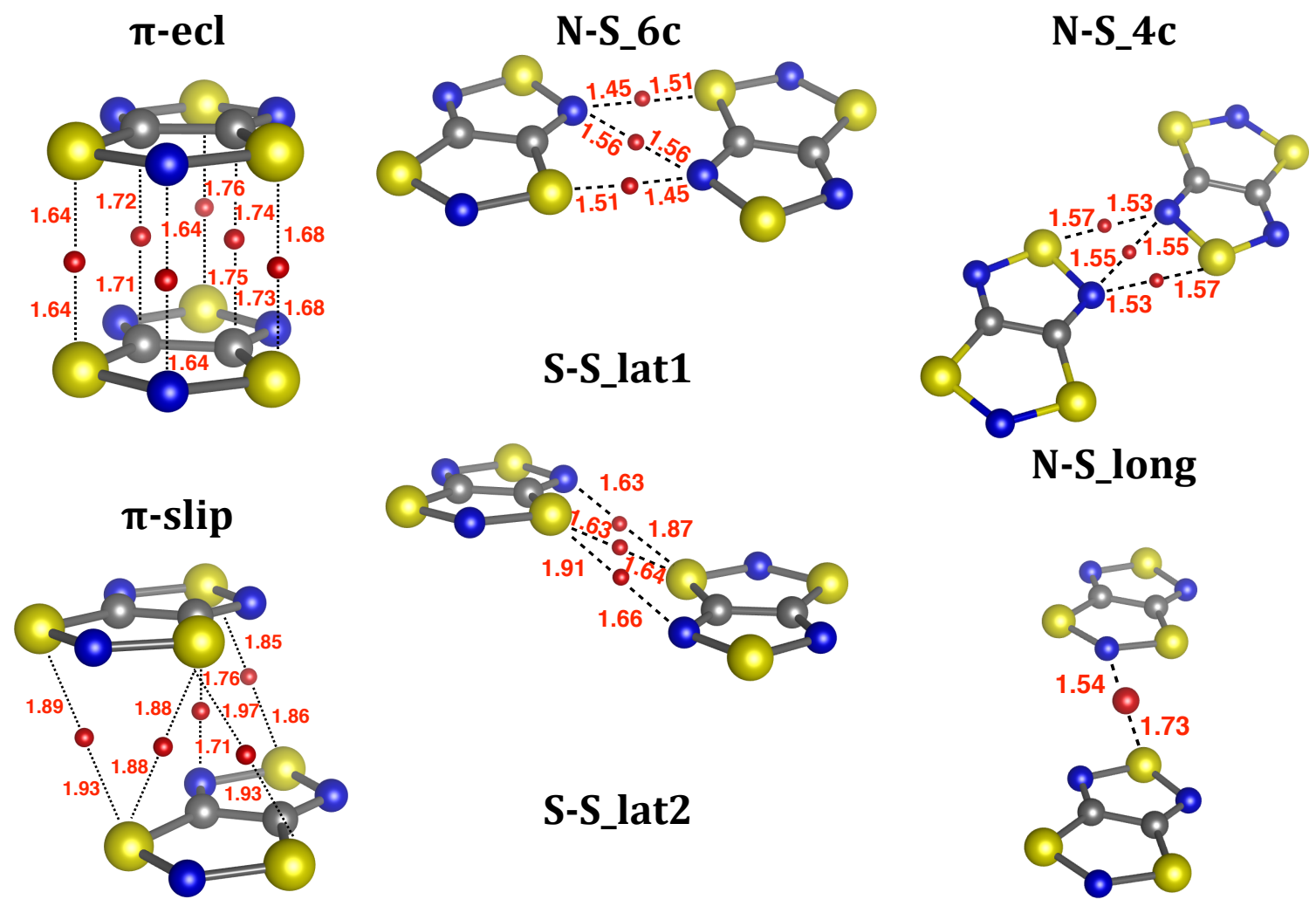

N-S_long
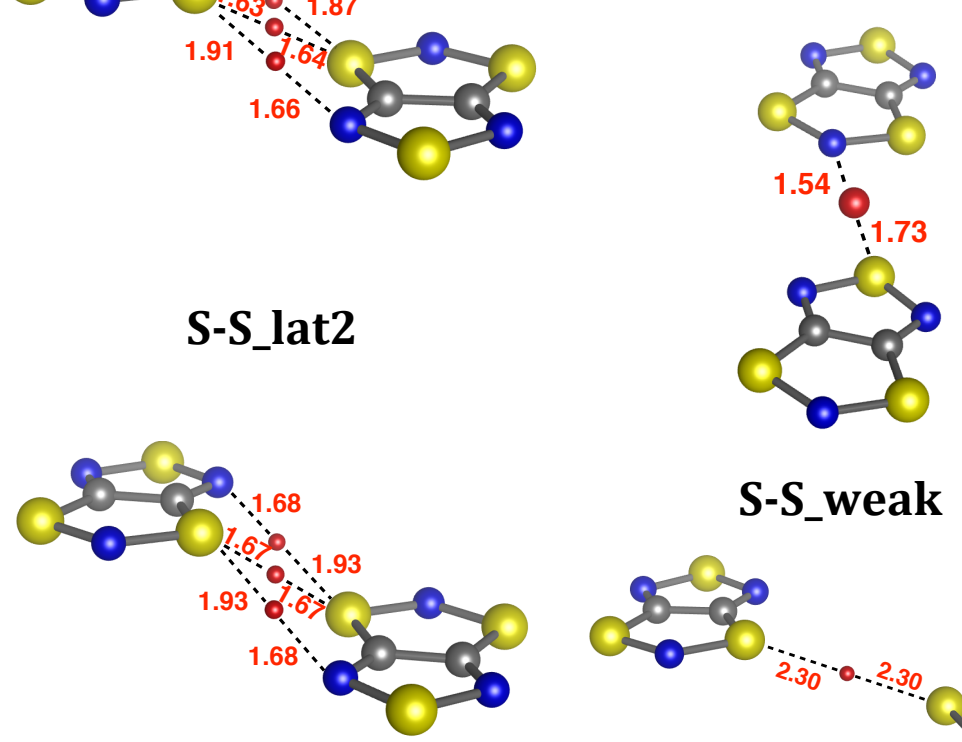

\section{S-S_weak}

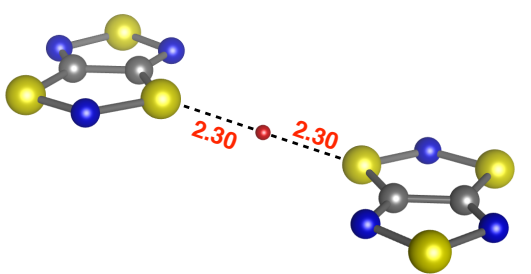

Figure 7. Bond critical points (BCP are marked as pink spheres) associated with several interatomic contacts of the different types of intermolecular interactions between radicals in the LT polymorph of TTTA. The images show the distances (given in $\AA$ ) between the bond critical points and the atoms.

The number of BCPs (Figures 7 and 8) and the electron density at these BCPs (Table 7) correlate well with the strength of interaction energies of the isolated pairs of radicals ( $c f$. Tables 2 and 3). The $\pi$-ecl interaction at LT, which is the most attractive interaction, features the largest number of BCPs (six), together with the largest value of the density on some of these critical points. In going from the $\pi$-ecl interaction of LT to the $\pi$-slip interaction of HT, not only the number of BCPs decreases (from six to five) but also the corresponding electron densities. 

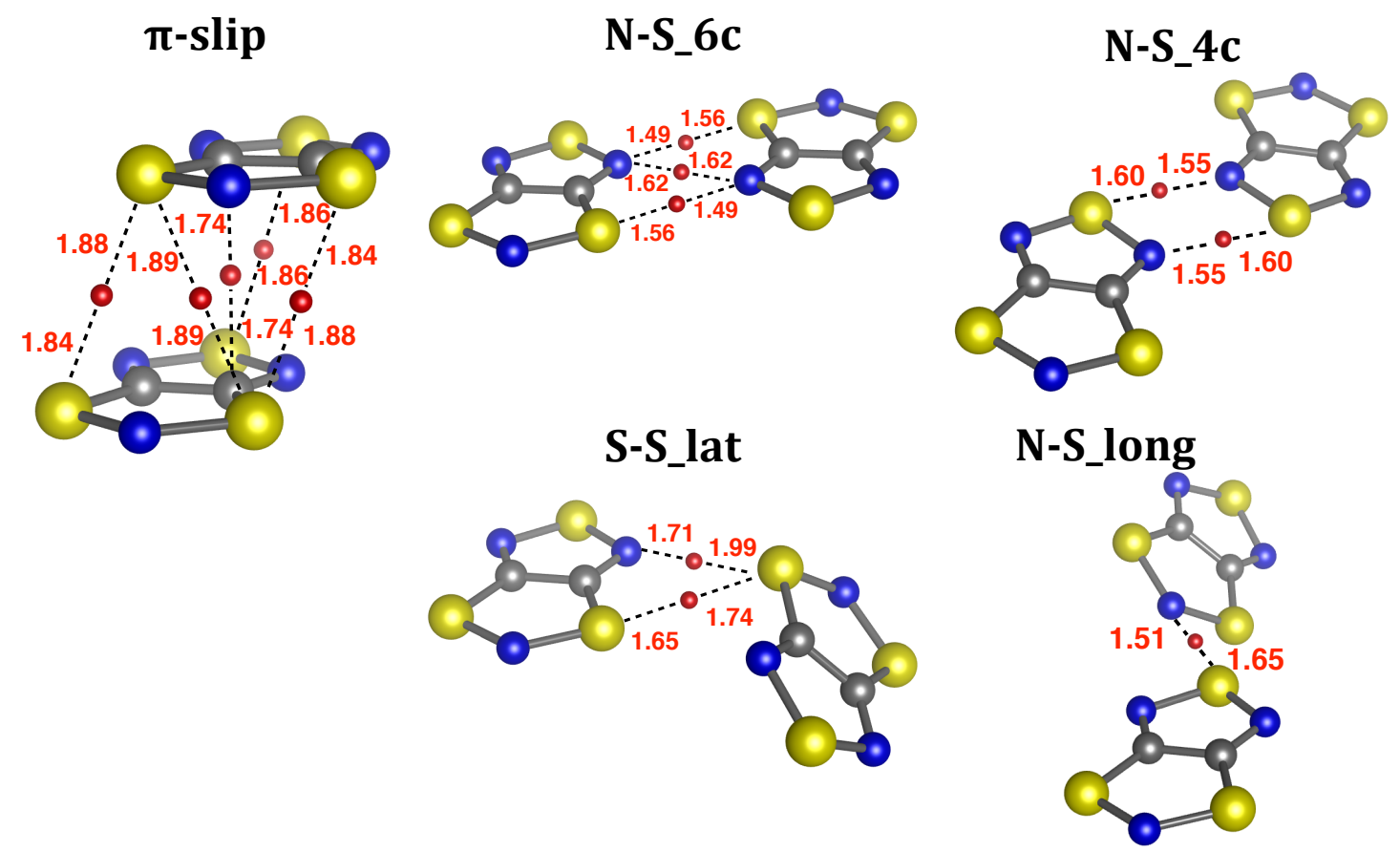

Figure 8. Bond critical points (BCP are marked as pink spheres) associated with several interatomic contacts of the different types of intermolecular interactions between radicals in the HT polymorph of TTTA. The images show the distances (given in $\AA$ ) between the bond critical points and the atoms.

Interestingly, the BCP between the nitrogen atoms of the dithiazolyl rings, which are the atoms that formally hold the unpaired electron of the radical, disappears upon transformation of the $\pi$-ecl(LT) interaction into the $\pi$-slip(HT) interaction. Among all lateral interactions, the N-S_6c type of interactions exhibit both the largest number of BCPs and the largest values of electron density, which is consistent with the fact that these are the strongest lateral interactions. The analysis of BCPs also reflects the strong weakening of the lateral S...S contact in going from the S-S_lat interaction in HT the to S-S_weak interaction in LT. Even though the elongated S...S contact in S-S_weak(LT) still features a BCP, the data in Table 7 shows that its associated value of the electron density is one order of magnitude smaller than the corresponding value in the BCP associated with the S...S contact in the S-S_lat(HT) interaction. It is worth mentioning that the

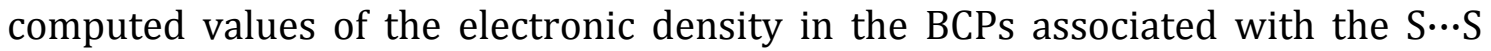

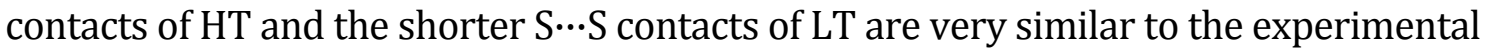
value determined for the $\mathrm{S} \cdots \mathrm{S}$ contacts in $\mathrm{TiS}_{2}{ }^{77}$ and the computed values for other organic crystals featuring S...S chalcogen interactions. ${ }^{78,79}$ 
Table 7. Values of the electronic density (in atomic units, i.e, $e \mathrm{a}_{0}{ }^{-3}$ ) at the bond critical points associated with the intermolecular contacts in the LT and HT polymorphs of TTTA. For each type of intermolecular interaction (see Figs. 6 and 7), the value of the electronic density is reported for all the interatomic contacts presenting a bond critical point. ${ }^{a}$

\begin{tabular}{|c|c|c|c|}
\hline \multicolumn{2}{|c|}{ HT } & \multicolumn{2}{|c|}{ LT } \\
\hline \multicolumn{2}{|c|}{$\pi$-slip } & \multicolumn{2}{|c|}{$\pi-e c l$} \\
\hline S(DTA) $\cdots S(D T A)$ & $0.0064^{b}$ & S(DTA) $\cdots S(D T A)$ & $0.0146^{b}$ \\
\hline $\mathrm{N}(\mathrm{DTA}) \cdots \mathrm{N}(\mathrm{DTA})$ & $-c$ & $\mathrm{~N}(\mathrm{DTA}) \cdots \mathrm{N}(\mathrm{DTA})$ & 0.0054 \\
\hline C(DTA) $\cdots C(\mathrm{DTA})$ & 0.0044 & C(DTA) $\cdots C(D T A)$ & $0.0045^{b}$ \\
\hline $\mathrm{S}(\mathrm{TDA}) \cdots \mathrm{S}(\mathrm{TDA})$ & 0.0064 & $\mathrm{~S}(\mathrm{TDA}) \cdots \mathrm{S}(\mathrm{TDA})$ & 0.0096 \\
\hline \multicolumn{2}{|c|}{ N-S_6c } & \multicolumn{2}{|c|}{ N-S 6c } \\
\hline $\mathrm{N}(\mathrm{TDA}) \cdots \mathrm{S}(\mathrm{DTA})$ & $0.0112^{b}$ & $\mathrm{~N}(\mathrm{TDA}) \cdots \mathrm{S}(\mathrm{DTA})$ & $0.0134^{b}$ \\
\hline $\mathrm{N}(\mathrm{TDA}) \cdots \mathrm{N}(\mathrm{TDA})$ & 0.0054 & $\mathrm{~N}(\mathrm{TDA}) \cdots \mathrm{N}(\mathrm{TDA})$ & 0.0067 \\
\hline \multicolumn{2}{|c|}{ N-S_4c } & \multicolumn{2}{|c|}{ N-S_4c } \\
\hline $\mathrm{N}(\mathrm{TDA}) \cdots \mathrm{S}(\mathrm{TDA})$ & $0.0088^{b}$ & $\mathrm{~N}(\mathrm{TDA}) \cdots \mathrm{S}(\mathrm{TDA})$ & $0.0095^{b}$ \\
\hline $\mathrm{N}(\mathrm{TDA}) \cdots \mathrm{N}(\mathrm{TDA})$ & - & $\mathrm{N}(\mathrm{TDA}) \cdots \mathrm{N}(\mathrm{TDA})$ & 0.0074 \\
\hline \multicolumn{2}{|c|}{ N-S_long } & \multicolumn{2}{|c|}{ N-S_long } \\
\hline $\mathrm{N}(\mathrm{DTA}) \cdots \mathrm{S}(\mathrm{TDA})$ & 0.0092 & $\mathrm{~N}(\mathrm{DTA}) \cdots \mathrm{S}(\mathrm{TDA})$ & 0.0079 \\
\hline \multicolumn{2}{|c|}{ S-S_lat } & \multicolumn{2}{|c|}{ S-S weak } \\
\hline$S(D T A) \cdots S(D T A)$ & 0,0093 & S(DTA) $\cdots$ S(DTA) & 0.0008 \\
\hline \multirow[t]{4}{*}{ S(DTA) $\cdots$ N(TDA) } & 0.0038 & S(DTA) $\cdots$ N(TDA) & - \\
\hline & & \multicolumn{2}{|c|}{ S-S_lat $1 /$ S-S_lat 2} \\
\hline & & $\mathrm{S}(\mathrm{DTA}) \cdots \mathrm{S}(\mathrm{DTA})$ & 0.0106 \\
\hline & & S(DTA) $\cdots N(T D A)$ & $0.0047^{b}$ \\
\hline
\end{tabular}

a The interatomic contacts are identified on the basis of the atoms of the heterocyclic rings (DTA and TDA refer to the dithiazolyl and thiadiazole rings, respectively) involved in the contact.

$\mathrm{b}$ This value results from taking the average of the density values of different bond critical points of the same type (see Figures 6 and 7).

c There is no critical point associated with this contact.

The evaluation of the nature of the key intermolecular interactions behind the different stability of the two polymorphs of TTTA was carried out by means of an energy decomposition analysis (EDA). Since the difference in stability between polymorphs is primarily dictated by the energy difference between the $\pi$-ecl(LT) and $\pi$-slip(HT) interactions and the energy difference between the S-S_weak(LT) and S-S_lat(HT) interactions, the EDA was carried out for these types of interaction only.

A comparison between the interaction energy components of the $\pi$-ecl dimers of LT and the $\pi$-slip dimers of HT reveals that all the attractive components of the interaction energy of the former are larger than those of the latter. The largest differences are found for the polarization and dispersion components ( $c f$. Tables 8 and 
Table 8. Energy decomposition analysis of the interaction energy of $\pi$-slip and $\pi$-ecl-type interactions in TTTA. All the components of the interaction energy are given in kcal mol-1.

\begin{tabular}{l|ccc} 
& $\pi$-slip (HT) & $\pi$-slip (LT) & $\pi$-ecl (LT) \\
\hline Electrostatic & -3.23 & -3.03 & -7.30 \\
Exchange & -2.74 & -2.46 & -6.35 \\
Repulsion & 15.28 & 14.09 & 33.07 \\
Polarization & -3.95 & -3.55 & -11.46 \\
DFT dispersion $^{\mathrm{a}}$ & -5.77 & -5.32 & -10.81 \\
Grimme dispersion $^{\mathrm{b}}$ & -5.63 & -5.47 & -6.59 \\
Total dispersion $^{\mathrm{c}}$ & -11.40 & -10.79 & -17.40
\end{tabular}

a This accounts for the dispersion that the PBE functional is able to recover by itself.

b This accounts for the dispersion recovered by Grimme's D2 semiempirical approach.

cThe values reported in this entry are just the sum of the values of "DFT dispersion" and "Grimme dispersion".

Table 9. Difference between the various components of the interaction energy of $\pi$-slip and $\pi$-ecl-type interactions in TTTA. All the differences are given in $\mathrm{kcal} \mathrm{mol}^{-1}$.

\begin{tabular}{l|cc} 
& $E(\pi-s l i p$, HT $)-E(\pi-e c l, L T)$ & $E(\pi-s l i p, H T)-E(\pi-s l i p, L T)$ \\
\hline Electrostatic & 4.07 & -0.20 \\
Exchange & 3.61 & -0.28 \\
Repulsion & -17.79 & 1.19 \\
Polarization & 7.51 & -0.40 \\
DFT dispersion & 5.04 & -0.45 \\
Grimme dispersion & 0.96 & -0.16 \\
Total dispersion & 6.00 & -0.61
\end{tabular}

Table 10. Energy decomposition analysis of the interaction energy of S-S_lat and S-S_weak type interactions in TTTA. All the components of the interaction energy are given in kcal $\mathrm{mol}^{-1}$.

\begin{tabular}{l|ccc} 
& S-S_lat (HT) & S-S_weak (LT) & $\Delta \mathrm{E}^{\mathrm{a}}$ \\
\hline Electrostatic & -1.61 & 0.02 & -1.63 \\
Exchange & -1.66 & -0.12 & -1.54 \\
Repulsion & 7.38 & 0.10 & 7.28 \\
Polarization & -2.81 & -0.36 & -2.45 \\
DFT dispersion $^{\mathrm{b}}$ & -2.12 & 0.04 & -2.16 \\
Grimme dispersion & -1.83 & -0.39 & -1.44 \\
Total dispersion & -3.95 & -0.35 & -3.60
\end{tabular}

a Given a component of the interaction energy, this column collects the difference between the value of this component in S-S_lat(HT) and in S-S_weak(LT).

$\mathrm{b}$ This accounts for the dispersion that the PBE functional is able to recover by itself.

9), in line with the known fact that pancake bonding between $\pi$-radicals is dominated by SOMO-SOMO bonding interaction and dispersion interaction. ${ }^{34,38,80}$. It is also worth mentioning that the electrostatic interaction between radicals is attractive and significantly larger in the $\pi$-ecl configuration even if in this configuration the 
polarized charges of the same sign are on top of each other. The repulsion energy between radicals, in turn, is much larger in the $\pi$-ecl dimers than in the $\pi$-slip dimers. Therefore, the $\pi$-ecl dimers of LT are more stable than the $\pi$-slip dimers of HT because the larger attractive components of the interaction energy (of which, the polarization and dispersion are the dominant ones) compensate the increase in repulsion energy in going from $\pi$-ecl(LT) to $\pi$-slip(HT). On the other hand, the values gathered in Table 10 demonstrate that the weakening of the lateral S $\cdots \mathrm{S}$ bonds in going from HT to LT is due to a weakening of all the attractive components of the interaction energy, especially the dispersion and polarization energies.

\section{Conclusions}

On the basis of the computational study herein presented, we have identified the intermolecular interactions that drive the crystal packing of the two polymorphs of TTTA and the intermolecular interactions that play a prime role in establishing the different stability of the polymorphs of this prototypical example of a bistable material based on dithiazolyl radicals. The formation of $\pi$-stacks of radicals in the two polymorphs is driven by strong $\pi-\pi$ interactions, which are the strongest of all intermolecular interactions between TTTA radicals. Among the lateral interactions that connect the $\pi$-stacks together, the stronger ones are those mediated by sixcenter $\mathrm{S} \cdots \mathrm{N}$ bridges, followed by those mediated by four-center $\mathrm{S} \cdots \mathrm{N}$ bridges.

The difference in stability between polymorphs is primarily governed by two types of intermolecular interactions: the $\pi-\pi$ intermolecular interactions along the $\pi$ stacks and the S $\cdots S$ lateral contacts between $\pi$-stacks. The $\pi-\pi$ interactions result in a substantial stabilization of the LT phase (relative to the HT phase) because the binding between radicals in the eclipsed $\pi$-dimers of the LT phase is stronger than in the slipped $\pi$-dimers of the HT phase. The stronger binding of the eclipsed $\pi$ dimers mainly originates in larger values of the polarization and dispersion

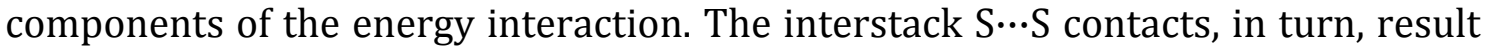
in a destabilization of the LT phase (relative to the HT phase) because some of the lateral S...S contacts of LT have a significantly weaker interaction than the lateral

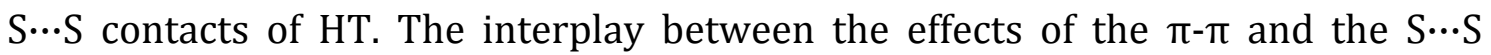


interactions (in which the effect of the $\pi-\pi$ interactions is the dominant one) in defining the difference in energy between polymorphs is tuned to a small but nonnegligible extent by other types of interstack interactions, including those interactions mediated by six- and four-center $\mathrm{S} \cdots \mathrm{N}$ bridges.

The results herein presented are not only relevant for the protypical TTTA compound, but also for other members of the family of dithiazolyl-based switchable materials because many of the supramolecular motifs identified as being important for driving the crystal packing and/or determining the difference in stability between polymorphs of TTTA are common to other members of this family. ${ }^{12,14-16,23}$ Therefore, our results provide valuable information for the rationalization of the crystal packing and spin transition temperatures of DTAbased switchable crystals, as well as for the design of new materials of this type.

\section{Acknowledgements}

FM, MD, JJN and JRA acknowledge financial support from MINECO through Grant CTQ2017-87773-P/AEI/FEDER, UE and Spanish Structures Excellence María de Maeztu program through grant MDM-2017-0767. FM, MD, JJN and JRA are also thankful to the Catalan DURSI (2017SGR348 grant) and to BSC and CSUC for the allocation of computer time. TF acknowledges funding from the European Union's Horizon 2020 research and innovation programme under the Marie Curie Skłodowska-Curie grant agreement no. 642294. 


\section{References}

${ }^{1}$ Rawson, J. M.; Alberola, A.; Whalley, A. "Thiazyl radicals: old materials for new molecular devices", J. Mater. Chem. 2006, 16, 2560-2575.

2 Preuss, K. E. “Metal complexes of thiazyl radicals”, Dalton Trans., 2007, 2357-2369.

${ }^{3}$ Hicks, R. G. "What's new in stable radical chemistry?", Org. Biomol. Chem., 2007, 5, 1321-1338.

${ }^{4}$ Hicks, R.G. in Stable Radicals: Fundamental and Applied Aspects of odd-electron compounds, R.G. Hicks, Ed. Wiley, New York 2010, p317-380.

${ }^{5}$ Lahti, P. M. "Structure-property relationships for metal-free organic materials", Adv. Phys. Org. Chem., 2011, 45, 93-169.

${ }^{6}$ Ratera, I.; Veciana, J. "Playing with organic radicals as building blocks for functional molecular materials", Chem. Soc. Rev., 2012, 41, 303-349.

7 Winter, S. M.; Hill, S.; Oakley, R. T. "Magnetic Ordering and Anisotropy in Heavy Atom Radicals", J. Am. Chem. Soc., 2015, 137, 3720-3730.

${ }^{8}$ Mailman, A.; Wong, J. W.; Winter, S. M.; Claridge, R. C.; Robertson, C. M.; Assoud, A.; Yong, W.; Steven, E.; Dube, P. A.; Tse, J. S. "Fine Tuning the Performance of Multiorbital Radical Conductors by Substituent Effects", J. Am. Chem. Soc., 2017, 139, 1625-1635.

${ }^{9}$ Sato, 0. "Dynamic molecular crystals with switchable physical properties", Nat. Chem., 2016, 8, 644-656.

${ }^{10}$ Bousseksou, A.; Molnár, G.; Salmon, L.; Nicolazzi, W. "Molecular spin crossover phenomenon: recent achievements and prospects", Chem. Soc. Rev. 2011, 40, 3313-3335.

${ }^{11}$ Spin-Crossover Materials-Properties and Applications, (Ed. Halcrow, M. A.), Wiley, Hoboken, 2013.

12 Barclay, T. M.; Cordes, A. W.; George, N. A.; Haddon, R. C.; Itkis, M. E.; Mashuta, M. S.; Oakley, R. T.; Patenaude, G. W.; Reed, R. W.; Richardson, J. F. "Redox, Magnetic, and Structural Properties of 1,3,2-Dithiazolyl Radicals. A Case Study on the Ternary Heterocycle $\mathrm{S}_{3} \mathrm{~N}_{5} \mathrm{C}_{4}$ ", J. Am. Chem. Soc., 1998, 120, 352-360.

${ }^{13}$ Fujita, W.; Awaga, K., "Room-temperature magnetic bistability in organic radical crystals”, Science 1999, 286, 261-262.

${ }^{14}$ Brusso, J. L.; Clements, O. P.; Haddon, R. C.; Itkis, M. E.; Leitch, A. A.; Oakley, R. T.; Reed, R. W.; Richardson, J. F. "Bistabilities in 1,3,2-Dithiazolyl Radicals", J. Am. Chem. Soc. 2004, 126, 8256-8265. 
15 Brusso, J. L.; Clements, O. P.; Haddon, R. C.; Itkis, M. E.; Leitch, A. A.; Oakley, R. T.; Reed, R. W.; Richardson, J. F. "Bistability and the Phase Transition in 1,3,2Dithiazolo[4,5-b]pyrazin-2-yl”, J. Am. Chem. Soc., 2004, 126, 14692-14693.

16 Alberola, A.; Collis, R. J.; Humphrey, S. M.; Less, R. J.; Rawson, J. M. "Spin transitions in a dithiazolyl radical: preparation, crystal structures, and magnetic properties of 3-cyanobenzo-1,3,2-dithiazolyl, $\mathrm{C}_{7} \mathrm{H}_{3} \mathrm{~S}_{2} \mathrm{~N}_{2}$ ", Inorg. Chem., 2006, 45, 1903-1905.

17 Lekin, K.; Winter, S. M.; Downie, L. E.; Bao, X.; Tse, J. S.; Desgreniers, S.; Secco, R. A.; Dube, P. A.; Oakley, R. T. "Hysteretic Spin Crossover between a Bisdithiazolyl Radical and Its Hypervalent $\sigma$-Dimer", J. Am. Chem. Soc., 2010, 132, 16212-16224.

${ }^{18}$ Alberola, A.; Eisler, D. J.; Harvey, L.; Rawson, J. M. "Molecular tailoring of spintransition materials: preparation, crystal structure and magnetism of trifluoromethyl-pyridyl-1,3,2-dithiazolyl", CrystEngComm 2011, 13, 1794-1796.

${ }^{19}$ Rawson, J. M.; Hayward, J. in Spin-Crossover Materials: Properties and Applications, (Ed. M. A. Halcrow), Wiley, Hoboken, 2013, pp. 225-237.

${ }^{20}$ Phan, H.; Lekin, K.; Winter, S. M.; Oakley, R. T.; Shatruk, M. "Photoinduced Solid State Conversion of a Radical $\sigma$-Dimer to a $\pi$-Radical Pair", J. Am. Chem. Soc., 2013, 135, 15674-15677.

${ }^{21}$ Lekin, K.; Phan, H.; Winter, S. M.; Wong, J. W.; Leitch, A. A.; Laniel, D.; Yong, W.; Secco, R. A.; Tse, J. S.; Desgreniers, S. "Heat, Pressure and Light-Induced Interconversion of Bisdithiazolyl Radicals and Dimers", J. Am. Chem. Soc., 2014, 136, 8050-8062.

22 Vela, S.; Reardon, M. B.; Jakobsche, C. E.; Turnbull, M. M.; Ribas-Arino, J.; Novoa, J. J. "Bistability in Organic Magnetic Materials: A Comparative Study of the Key Differences between Hysteretic and Non-hysteretic Spin Transitions in Dithiazolyl Radicals", Chem. Eur. J., 2017, 23, 3479-3489.

${ }^{23}$ Bates, D.; Robertson, C. M.; Leitch, A. A.; Dube, P. A.; Oakley, R. T. "Magnetic Bistability in Naphtho-1,3,2-dithiazolyl: Solid State Interconversion of a Thiazyl $\pi-$ Radical and Its N-N $\sigma$-Bonded Dimer", J. Am. Chem. Soc., 2018, 140, 3846-3849.

${ }^{24}$ Matsuzaki, H.; Fujita, W.; Awaga, K.; Okamoto, H. "Photoinduced PhaseTransition in an Organic Radical Crystal with Room-Temperature Optical and Magnetic Bistability", Phys. Rev. Lett., 2003, 91, 017403.

${ }^{25}$ Suizu, R.; Iwasaki, A.; Shuku, Y.; Awaga, K. "Spatially inhomogeneous, stepwise phase transitions in a thiazyl diradical: a structural mismatch induced by lattice transformation", J. Mater. Chem. C, 2015, 3, 7968-7977. 
${ }^{26}$ Fatila, E. M.; Mayo, R. A.; Rouzieres, M.; Jennings, M. C.; Dechambenoit, P.; Soldatov, D. V.; Mathoniere, C.; Clerac, R.; Coulon, C.; Preuss, K. E. "Radical-Radical Recognition: Switchable Magnetic Properties and Re-entrant Behavior", Chem. Mater., 2015, 27, 4023-4032.

27 Brooker, S. "Spin crossover with thermal hysteresis: practicalities and lessons learnt”, Chem. Soc. Rev. 2015, 44, 2880-2892

${ }^{28}$ T Romero-Morcillo, T.; Seredyuk, M.; Muñoz, M. C.; Real, J. A. "Meltable Spin Transition Molecular Materials with Tunable $\mathrm{T}_{\mathrm{c}}$ and Hysteresis Loop Width", Angew. Chem. Int. Ed., 2015, 54, 14777-14781.

${ }^{29}$ Gómez, V.; Sáenz de Pipaón, C.; Maldonado-Illescas, P.; Waerenborgh, J. C.; Martin, E.; Benet-Buchholz, J.; Galán-Mascarós, J. R. "Easy Excited-State Trapping and Record High $\mathrm{T}_{\text {TIESST }}$ in a Spin-Crossover Polyanionic Fe ${ }^{\mathrm{II}}$ Trimer "J. Am. Chem. Soc. 2015, 137, 11924-11927.

30 Itkis, M. E., Chi, X., Cordes, A.W., Haddon, R. C. "Magneto-Opto-Electronic Bistability in a Phenalenyl-Based Neutral Radical", Science, 2002, 296, 1443-1445.

${ }^{31}$ Hicks, R.G. "A new spin on bistability”, Nat. Chem. 2011, 3, 189-191.

${ }^{32}$ Li, T.; Tan, G.; Shao, D.; Li, J.; Zhang, Z.; Song, Y.; Sui, Y.; Chen, S.; Fang, Y.; Wang, X. "Magnetic Bistability in a Discrete Organic Radical", J. Am. Chem. Soc., 2016, 138, 10092-10095.

${ }^{33}$ Kahn, O., Martinez, C. J. "Spin-transition polymers: from molecular materials toward memory devices", Science, 1998, 279, 44-48.

34 Miller, J. S., Novoa, J. J. "Four-center carbon-carbon bonding”, Acc. Chem. Res., 2007, 40, 189-196.

35 Jose, D.; Datta, A. "Role of multicentered bonding in controlling magnetic interactions in $\pi$-stacked bis-dithiazolyl radical", Cryst. Growth Des., 2011, 11, 3137-3140.

${ }^{36}$ Mulliken, R. S., Person, W. B. Molecular Complexes, Wiley, Hoboken 1969, Chapter 16.

37 Preuss, K. E. "Pancake bonds: pi-Stacked dimers of organic and light-atom radicals" Polyhedron 2014, 79, 1-15 .

38 Cui, Z.; Lischka, H.; Beneberu, H. Z. and Kertesz, M. "Double Pancake Bonds: Pushing the Limits of Strong $\pi-\pi$ Stacking Interactions", J. Am. Chem. Soc., 2014, 136, 12958-12965. 
${ }^{39}$ Beneberu, H. Z.; Tian, Y.-H.; Kertesz, M. "Bonds or not bonds? Pancake bonding in 1,2,3,5-dithiadiazolyl and 1,2,3,5-diselenadiazolyl radical dimers and their derivatives", Phys. Chem. Chem. Phys., 2012, 14, 10713-10725.

${ }^{40}$ Clarke, C. S.; Jornet-Somoza, J.; Mota, F.; Novoa, J. J.; Deumal, M. "Origin of the Magnetic Bistability in Molecule-Based Magnets: A First-Principles Bottom-Up Study of the TTTA Crystal", J. Am. Chem. Soc., 2010, 132, 17817-17830.

41 Francese, T.; Ribas-Arino, J.; Novoa, J. J.; Havenith, R. W. A.; Broer, R.; de Graaf, C.; Deumal, M. "The magnetic fingerprint of dithiazolyl-based molecule magnets", Phys. Chem. Chem. Phys., 2018, 20, 20406-20416.

${ }^{42}$ Note that both vibrational energy and entropy might play a non-negligible role. However, this is beyond the scope of the present manuscript.

43 Wolmershäuser, G.; Johann, R. "1,3,5-trithia-2,4,6-triazapentalenyl-a stable sulfurnitrogen radical”, Angew. Chem., Int. Ed. 1989, 28, 920-921.

${ }^{44}$ McManus, G. D.; Rawson, J. M.; Feeder, N.; van Duijn, J.; McInnes, E. J. L.; Novoa, J. J.; Burriel, R.; Palacio, F.; Oliete, P. "Synthesis, crystal structures, electronic structure and magnetic behaviour of the trithiatriazapentalenyl radical, $\mathrm{C}_{2} \mathrm{~S}_{3} \mathrm{~N}_{3}$ ", J. Mater. Chem. 2001, 11, 1992-2003.

${ }^{45}$ Fujita, W.; Awaga, K.; Matsuzaki, H.; Okamoto, H. "Room-temperature magnetic bistability in organic radical crystals: paramagnetic-diamagnetic phase transition in 1,3,5-trithia-2,4,6-triazapentalenyl", Phys. Rev. B 2002, 65, 064434-1-9.

46 Tanaka, T.; Fujita, W.; Awaga, K. "Pressure effects on magnetic bistability in a heterocyclic thiazyl radical TTTA", Chem. Phys. Lett. 2004, 393, 150-152.

47 Takahashi, Y.; Suemoto, T.; Oguri, S.; Takeda, J. "Relaxation processes from charge-transfer excited states of organic radical 1,3,5-trithia-2,4,6triazapentalenyl crystals studied by ultrafast luminiscence spectroscopy", Phys. Rev. B, 2006, 74, 193104-1-4.

48 Furuya, M.; Kawazoe, Y.; Ohno, K. "Ab initio study on geometrical structures of the TTTA molecular crystal”, Sci. Tech. Adv. Mater., 2004, 5, 689-692.

49 Ohno, K.; Noguchi, Y.; Yokoi, T.; Ishii, S.; Takeda, J.; Furuya, M. "Significant reduction of on-site Coulomb energy $U$ due to short-range correlation in an organic Mott Insulator", ChemPhysChem 2006, 7, 1820-1824.

${ }^{50}$ Naumov, P.; Hill, J. P.; Sakurai, K.; Tanaka, M.; Ariga, K. "Structural study of the thermally induced and photoinduced phase transitions of the 1,3,5-trithia- 2,4,6triazapentalenyl (TTTA) radical", J. Phys. Chem. A 2007, 111, 6449-6455. 
51 Kon, T.; Oguri, S.; Katayama, I.; Kodaira, T.; Takeda, J. "Highly efficient photoinduced phase transition in an organic radical crystal via two-photon absorption process", Phys. Rev. B 2009, 79, 035106-1-4.

52 Katayama, I.; Kon, T.; Mitarai, K.; Takeda, J. "Nonlinear conversion dynamics from self-trapped exciton states to a macroscopic photoinduced phase in strongly correlated organic radical crystals", Phys. Rev. B, 2009, 80, 092103-1-4.

${ }^{53}$ Domingo, A.; Vérot, M.; Mota, F.; de Graaf, C.; Novoa, J. J.; Robert, V. "Impact of short and long-range effects on the magnetic interactions in neutral organic radical-based materials", Phys. Chem. Chem. Phys., 2013, 15, 6982-6989.

${ }^{54}$ Vela, S.; Mota, F.; Deumal, M.; Suizu, R.; Shuku, Y.; Mizuno, A.; Awaga, K.; Shiga, M.; Novoa, J. J.; Ribas-Arino, J. "The key role of vibrational entropy in the phase transitions of dithiazolyl-based bistable magnetic materials", Nat. Commun. 2014, 5,4411 .

55 Vela, S.; Deumal, M.; Shiga, M.; Novoa, J. J.; Ribas-Arino, J. “Dynamical effects on the magnetic properties of dithiazolyl bistable materials", Chem. Sci., 2015, 6, 2371-2381.

${ }^{56}$ Perdew, J. P.; Burke, K.; Ernzerhof, M. "Generalized Gradient Approximation Made Simple”, Phys. Rev. Lett., 1996, 77, 3865-3868.

57 Perdew, J. P.; Burke, K.; Ernzerhof, M. "Generalized Gradient Approximation Made Simple (Errata)”, Phys. Rev. Lett., 1996, 78, 1396.

58 Vanderbilt, D. "Soft self-consistent pseudopotentials in a generalized eigenvalue formalism”, Phys. Rev. B, 1990, 41, 7892-7985.

${ }^{59}$ QUANTUM ESPRESSO, Version 5.4.0., Giannozzi, P.; Andreussi, O.; Brumme, T.; Bunau, O.; Nardelli, M. B.; Calandra, M.; Car, R.; Cavazzoni, C.; Ceresoli, D.; Cococcioni, M.; Colonna, N.; Carnimeo, I.; Corso, A. D.; Gironcoli, S. de; Delugas, P.; Jr, R. A. D.; Ferretti, A.; Floris, A.; Fratesi, G.; Fugallo, G.; Gebauer, R.; Gerstmann, U.; Giustino, F.; Gorni, T.; Jia, J.; Kawamura, M.; Ko, H.-Y.; Kokalj, A.; Küçükbenli, E.; Lazzeri, M.; Marsili, M.; Marzari, N.; Mauri, F.; Nguyen, N. L.; Nguyen, H.-V.; Oterode-la-Roza, A.; Paulatto, L.; Poncé, S.; Rocca, D.; Sabatini, R.; Santra, B.; Schlipf, M.; Seitsonen, A. P.; Smogunov, A.; Timrov, I.; Thonhauser, T.; Umari, P.; Vast, N.; Wu, X.; Baroni, S. "Advanced capabilities for materials modelling with Quantum ESPRESSO”, J.Phys.:Condens.Matter., 2017, 29, 465901.

${ }^{60}$ Grimme, S. "Semiempirical GGA-Type Density Functional Constructed with a Long-Range Dispersion Correction", J. Comput. Chem., 2006, 27, 1787-1799.

61 Dunning, T. H. "Gaussian basis sets for use in correlated molecular calculations. I. The atoms boron through neon and hydrogen", J. Chem. Phys., 1989, 90, 10071023. 
62 Woon, D. E.; Dunning, T. H. “Gaussian basis sets for use in correlated molecular calculations. III. The atoms aluminum through argon", J. Chem. Phys., 1993, 98, 1358-1371.

63 Dovesi, R.; Saunders, V. R.; Roetti, C.; Orlando, R.; Zicovich-Wilson, C. M.; Pascale, F.; Civalleri, B.; Doll, K.; Harrison, N. M.; Bush, I. J.; D’Arco, P.; Llunell, M.; Llunell. CRYSTAL09 User's Manual. University of Torino: Torino, 2009.

${ }^{64}$ Dovesi, R.; Orlando, R.; Civalleri, B.; Roetti, C.; Saunders, V. R.; Zicovich-Wilson, C. M. "CRYSTAL: a computational tool for the ab initio study of the electronic properties of crystals", Z. Kristallogr., 2005, 220, 571-573.

${ }^{65}$ Angeli, C.; Cimiraglia, R.; Evangelisti, S.; Leininger, T.; Malrieu, J.-P. "Introduction of $n$-electron valence states for multireference perturbation theory", J. Chem. Phys., 2001, 114, 10252-10264.

${ }^{66}$ Angeli, C.; Cimiraglia, R.; Malrieu, J.-P. "n-electron valence state perturbation theory: A spinless formulation and an efficient implementation of the strongly contracted and of the partially contracted variants", J. Chem. Phys., 2002, 117, 9138-9153.

${ }^{67}$ Neese, F. ORCA - An ab Initio, Density Functional and Semiempirical Program Package, Version 3.0; Max Planck Institute for Bioinorganic Chemistry: Müllheim, 2012.

68 Kitaura, K.; Morokuma, K. "A new energy decomposition scheme for molecular interactions within the Hartree-Fock approximation”, Int. J. Quantum Chem., 1976, 10, 325-331.

${ }^{69} \mathrm{Su}, \mathrm{P}$;; Li, H. "Energy decomposition analysis of covalent bonds and intermolecular interactions", J. Chem. Phys., 2009, 131, 014102.

${ }^{70}$ Schmidt, M. W.; Baldridge, K. K.; Boatz, J. A.; Elbert, S. T.; Gordon, M. S.; Jensen, J. H.; Koseki, S.; Matsunaga, N.; Nguyen, K. A.; Su, S.; Windus, T. L.; Dupuis, M.; Montgomery, J. A. "General atomic and molecular electronic structure system", J. Comput. Chem., 1993, 14, 1347-1363.

${ }^{71}$ Bader, R. Atoms in Molecules: A Quantum Theory. 1994, USA: Oxford University Press.

72 AIMAll (Version 17.11.14), Keith, T.A., TK Gristmill Software, Overland Park KS, USA, 2017 (aim.tkgristmill.com)

73 Gaussian 09, Revision C.01, Frisch, M. J.; Trucks, G. W.; Schlegel, H. B.; Scuseria, G. E.; Robb, M. A.; Cheeseman, J. R.; Scalmani, G.; Barone, V.; Mennucci, B.; Petersson, G. A.; Nakatsuji, H.; Caricato, M.; Li, X.; Hratchian, H. P.; Izmaylov, A. F.; Bloino, J.; Zheng, G.; Sonnenberg, J. L.; Hada, M.; Ehara, M.; Toyota, K.; Fukuda, R.; Hasegawa, J.; Ishida, M.; Nakajima, T.; Honda, Y.; Kitao, O.; Nakai, H.; Vreven, T.; Montgomery, J. A., Jr.; Peralta, J. E.; Ogliaro, F.; Bearpark, M.; Heyd, J. J.; Brothers, E.; Kudin, K. N.; 
Staroverov, V. N.; Kobayashi, R.; Normand, J.; Raghavachari, K.; Rendell, A.; Burant, J. C.; Iyengar, S. S.; Tomasi, J.; Cossi, M.; Rega, N.; Millam, J. M.; Klene, M.; Knox, J. E.; Cross, J. B.; Bakken, V.; Adamo, C.; Jaramillo, J.; Gomperts, R.; Stratmann, R. E.; Yazyev, O.; Austin, A. J.; Cammi, R.; Pomelli, C.; Ochterski, J. W.; Martin, R. L.; Morokuma, K.; Zakrzewski, V. G.; Voth, G. A.; Salvador, P.; Dannenberg, J. J.; Dapprich, S.; Daniels, A. D.; Farkas, Ö.; Foresman, J. B.; Ortiz, J. V.; Cioslowski, J.; Fox, D. J. Gaussian, Inc., Wallingford CT, 2009.

74 The computed difference in lattice cohesive energy does not take into account neither the vibrational energy nor the entropy.

75 Thomas, S.P.; Spackman, P.R.; Jayatilaka, D.; Spackman, M.A. "Accurate lattice energies for molecular crystals from experimental crystal structures". J. Chem. Theory Comput. 2018, 14, 1614 - 1623.

${ }^{76}$ Note that the absolute value of $0.85 \mathrm{kcal} \mathrm{mol}^{-1}$ is per mol of TTTA radical, while the value of $-1.70 \mathrm{kcal} \mathrm{mol}^{-1}$ in Table 4 is per mol of a pair of TTTA radicals.

77 Kasai, H.; Tolborg, K.; Sist, M.; Zhang, J.; Hathwar, V.R.; Filsø, M.ø; Cenedese, S.; Sugimoto, K.; Overgaard, J.; Nishibori, E.; Iversen, B.B. "X-ray electron density investigation of chemical bonding in van der Waals materials", Nat. Mat. 2018, 17, $249-252$.

${ }^{78}$ Bai, M.; Thomas, S.P.; Kottokkaran, R.; Nayak, S. K.; Ramamurthy, P, C.; Guru Row, T. N. "A donor-acceptor-donor structured organic conductor with S $\cdots$ S chalcogen bonding", Cryst. Growth Des., 2014, 14, 459 - 466.

79 Thomas, S.P.; Sathishkumar, R.; Guru Row, T.N. "Organic alloys of room temperature liquids thiophenol and selenophenol", Chem. Commun. 2015, 51, 14255 - 14258.

${ }^{80}$ Jung, Y.; Head-Gordon, M. "What is the nature of the long bond in the $\mathrm{TCNE}_{2}{ }^{2-} \pi-$ dimer?”, Phys. Chem. Chem. Phys., 2004, 6, 2008 - 2011. 
FOR TABLE OF CONTENTS USE ONLY

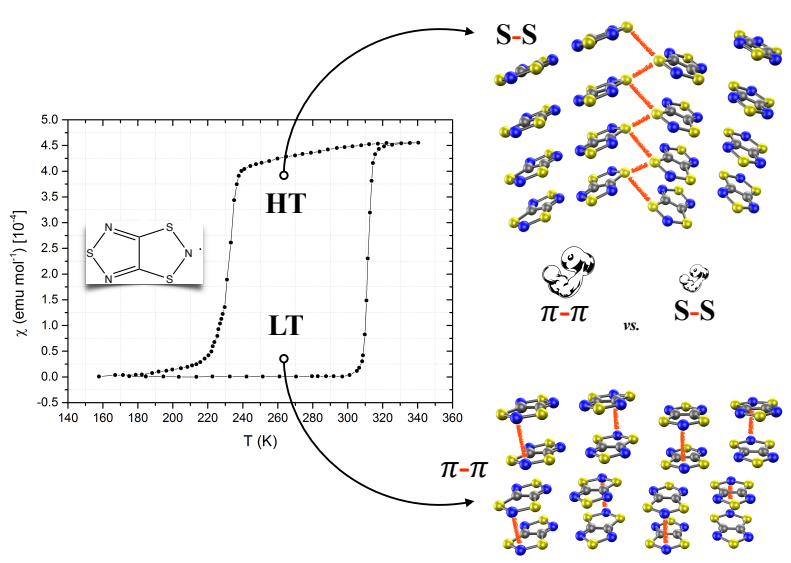

A computational study of the intermolecular interactions existing in the two polymorphs of the bistable neutral radical 1,3,5-trithia-2,4,6-triazapentalenyl (TTTA) has shown that the energy difference between its two phases is mainly controlled by an interplay between $\pi-\pi$ interactions and $S \cdots S$ interactions. 